\title{
Caspase-8 modulates physiological and pathological angiogenesis during retina development
}

\author{
Nathalie Tisch, ${ }^{1,2,3}$ Aida Freire-Valls, ${ }^{1,4}$ Rosario Yerbes, ${ }^{1,5}$ Isidora Paredes, ${ }^{1,2,3}$ Silvia La Porta, ${ }^{2,6}$ Xiaohong Wang, ${ }^{1}$ \\ Rosa Martín-Pérez, ${ }^{7,8}$ Laura Castro, ${ }^{1}$ Wendy Wei-Lynn Wong, ${ }^{9}$ Leigh Coultas, ${ }^{10,11}$ Boris Strilic, ${ }^{12}$ Hermann-Josef Gröne, ${ }^{13}$ \\ Thomas Hielscher, ${ }^{14}$ Carolin Mogler, ${ }^{15}$ Ralf H. Adams, ${ }^{16,17}$ Peter Heiduschka, ${ }^{18}$ Lena Claesson-Welsh, ${ }^{19}$ Massimiliano Mazzone, ${ }^{7,8}$ \\ Abelardo López-Rivas, ${ }^{5,20}$ Thomas Schmidt, ${ }^{4}$ Hellmut G. Augustin, ${ }^{2,6}$ and Carmen Ruiz de Almodovar, ${ }^{1,2,3}$ \\ 'Biochemistry Center, ${ }^{2}$ European Center for Angioscience (ECAS), ${ }^{3}$ Institute for Transfusion Medicine and Immunology, Medical Faculty Mannheim, and ${ }^{4}$ Department of General, Visceral and \\ Transplantation Surgery, Heidelberg University, Heidelberg, Germany. ${ }^{5}$ Centro Andaluz de Biología Molecular y Medicina Regenerativa (CABIMER), Consejo Superior de Investigaciones Científicas (CSIC), \\ Universidad de Sevilla and Universidad Pablo de Olavide, Sevilla, Spain. ${ }^{6}$ Division of Vascular Oncology and Metastasis, Cerman Cancer Research Center, Heidelberg, Cermany. ${ }^{7}$ Lab of Tumor Inflammation \\ and Angiogenesis, Center for Cancer Biology (VIB), Leuven, Belgium. ${ }^{8}$ Lab of Tumor Inflammation and Angiogenesis, Department of Oncology, Katholieke Universiteit Leuven, Leuven, Belgium. ${ }^{9}$ Institute \\ of Experimental Immunology, University of Zurich, Zurich, Switzerland. ${ }^{10}$ Development and Cancer Division, Walter and Eliza Hall Institute of Medical Research, Parkville, Australia. "Department of Medical \\ Biology, University of Melbourne, Melbourne, Australia. ${ }^{12}$ Department of Pharmacology, Max Planck Institute for Heart and Lung Research, Bad Nauheim, Germany. ${ }^{13}$ Department of Cellular and Molecular \\ Pathology and ${ }^{14}$ Division of Biostatistics, German Cancer Research Center, Heidelberg, Germany. ${ }^{15}$ Institute of Pathology, TUM School of Medicine, Technical University of Munich, Munich, Germany. \\ ${ }^{16}$ Department of Tissue Morphogenesis, Max Planck Institute for Molecular Biomedicine, Münster, Cermany. ${ }^{17}$ Faculty of Medicine and ${ }^{18}$ Research Laboratory, Department of Ophthalmology, University \\ Medical Center, University of Münster, Münster, Germany. ${ }^{19}$ Department of Immunology, Genetics and Pathology, Science for Life Laboratory, Uppsala University, Uppsala, Sweden. ${ }^{20}$ Centro de Investigación \\ Biomédica en Red-Oncología (CIBERONC), Carlos III Health Institute, Madrid, Spain.
}

\begin{abstract}
During developmental angiogenesis, blood vessels grow and remodel to ultimately build a hierarchical vascular network. Whether, how, cell death signaling molecules contribute to blood vessel formation is still not well understood. Caspase-8 (Casp-8), a key protease in the extrinsic cell death-signaling pathway, regulates cell death via both apoptosis and necroptosis. Here, we show that expression of Casp-8 in endothelial cells (ECs) is required for proper postnatal retina angiogenesis. ECspecific Casp-8-KO pups (Casp- $8^{\text {ЕСК) }}$ showed reduced retina angiogenesis, as the loss of Casp-8 reduced EC proliferation, sprouting, and migration independently of its cell death function. Instead, the loss of Casp- 8 caused hyperactivation of p38 MAPK downstream of receptor-interacting serine/threonine protein kinase 3 (RIPK3) and destabilization of vascular endothelial cadherin (VE-cadherin) at EC junctions. In a mouse model of oxygen-induced retinopathy (OIR) resembling retinopathy of prematurity (ROP), loss of Casp-8 in ECs was beneficial, as pathological neovascularization was reduced in Casp- $8^{\text {Еско }}$ pups. Taking these data together, we show that Casp- 8 acts in a cell death-independent manner in ECs to regulate the formation of the retina vasculature and that Casp-8 in ECs is mechanistically involved in the pathophysiology of ROP.
\end{abstract}

\section{Introduction}

Functional blood vessels are of vital importance. Impaired vessel formation contributes to many pathological situations, including ischemic and inflammatory disorders. Ischemic retinopathies are the main causes of severe visual impairment and sight loss in premature children, diabetic adults, and the elderly population. In particular, retinopathy of prematurity (ROP), a developmental ocular neovascular disease and the major cause of acquired blindness in preterm infants (1), is characterized by excessive angiogenesis, breakdown of the endothelial barrier, vascular leakage, edema, hemorrhages, retinal detachment, and compromised vision (1). Although certain strategies to block excessive angiogenesis exist (2), identification of other pathways that regulate physiological

Authorship note: NT, AFV, and RY share the first authorship position Conflict of interest: The authors have declared that no conflict of interest exists. Copyright: (๖) 2019, American Society for Clinical Investigation. Submitted: June 13, 2018; Accepted: August 20, 2019; Published: October 22, 2019. Reference information: / Clin Invest. 2019;129(12):5092-5107. https://doi.org/10.1172/JCl122767. and pathological vessel growth is of high interest in order to further develop better or complementary therapeutic treatments.

The formation of functional blood vessel networks starts with the initial formation of a primitive vascular plexus from which new blood vessels sprout, coordinately expand, and branch. Redundant vessel branches are then selectively removed by vessel pruning to ultimately establish a hierarchical vascular network $(3,4)$. Whereas apoptosis has been implicated in certain conditions of vessel remodeling (5-9) and in the regulation of capillary vessel diameter (10), it is not known whether other forms of cell death contribute to developmental angiogenesis or whether cell death signaling molecules have cell death-independent functions in endothelial cells (ECs).

Caspase-8 (Casp-8) is the initiator caspase of the extrinsic apoptosis pathway that activates the effector caspase-3 (11). Cellular FLICE inhibitory protein (c-FLIP), an inactive homolog of Casp-8 that lacks its catalytic activity, can heterodimerize with Casp-8, prevent its full activation, and thus inhibit apoptosis (11). The c-FLIP/Casp- 8 heterodimer still possesses a basal Casp- 8 activity necessary to inhibit a second type of programmed cell 
death, called necroptosis (a nonapoptotic cell death characterized by swelling and rupture of the cell membrane) $(12,13)$. To do so, Casp-8 cleaves the receptor-interacting serine/threonine protein kinase 3 (RIPK3) and therefore blocks the activation of the ultimate effector of necroptosis, the pseudokinase mixed-lineage kinase domain-like (MLKL), downstream of RIPK3 (13). Therefore, Casp- 8 is a central signaling node in the determination of whether a particular cell dies by apoptosis or necroptosis. In addition to its role in cell death signaling, Casp- 8 has been described as regulating cell death-independent processes, such as cell migration $(14,15)$ or proliferation (16), and acting as DNA-damage sensor induced by cell proliferation (17).

Deletion of Casp-8 in mice is embryonically lethal. Interestingly, Casp-8-KO mouse embryos die at midgestation, presenting a circulatory failure phenotype and damaged blood vessel capillaries (18-20). EC-specific KO of Casp-8 during embryonic development mimics the severity of the full KO (21), suggesting that Casp-8 in ECs is required for the formation of a proper vascular system. However, whether Casp-8 is also required at later time points during vessel development and whether it plays a cell death-dependent or -independent function remain unknown.

In this regard, cell death-dependent and -independent functions of RIPK3 have been described in pathological angiogenesis. In a mouse model of melanoma metastasis, it has been shown that RIPK3 is involved in tumor-induced EC necroptosis, but also in vessel permeability via activation of p38 $(22,23)$. Whether RIPK3 not only contributes to pathological vessel formation and function, but also to vessel stability in physiological conditions of angiogenesis, is so far unknown.

Here, using a conditional inducible EC-specific Casp-8-KO

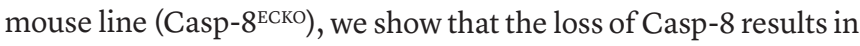
reduced postnatal retina angiogenesis. Most strikingly, we demonstrate that this phenotype is independent of necroptosis, as the loss of Casp-8 on a MLKL-null background still results in vascular defects. Instead, Casp- 8 regulates EC proliferation, sprouting, and migration as well as the stability of adherens and tight junctions. Mechanistically, we show that loss of Casp-8 leads to the constitutive phosphorylation of $\mathrm{p} 38$ MAPK via RIPK3 and to defects in vascular endothelial cadherin (VE-cadherin) subcellular localization. Applying the oxygen-induced retinopathy (OIR) model, a model that resembles the pathology of ROP, we show that the loss of Casp- 8 in ECs is beneficial during this developmental neovascular eye disease, as pathological neovascularization in Casp- $8^{\mathrm{ECKO}}$ mice is reduced.

\section{Results}

Postnatal deletion of Casp- 8 in ECs results in impaired angiogenesis in the retina. To explore the function of Casp- 8 during developmental angiogenesis in vivo, we generated inducible EC-specific Casp-8KO mice. To do so, we crossed the tamoxifen-inducible Cdh5(PAC)-CreERT2 mouse line (24), in which Cre-recombinase is expressed under control of the EC-specific Cdh-5 promoter, with Casp- $8^{\mathrm{fl} / \mathrm{fl}}$ mice. Consistent with previously published data (21), therefore validating our generated mouse line, KO of Casp- 8 in the endothelium during embryonic development resulted in yolk sac and embryo vascular defects as well as in increased embryonic lethality and defects in the yolk sac vasculature (Supplemental
Figure 1, A and B; supplemental material available online with this article; https://doi.org/10.1172/JCI122767DS1).

To analyze the effect of loss of Casp-8 in ECs during postnatal vascular development, we induced Cre recombination in newborn pups, as previously described (ref. 25 and Figure 1A), and checked the recombination efficiency in isolated lung ECs (Supplemental Figure 1, C and D). Tamoxifen treatment resulted in $70 \%$ reduc-

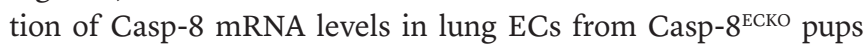
at P6 (Supplemental Figure 1D). In contrast to the lethal effects of loss of Casp-8 in the embryonic vasculature, postnatal KO of Casp- 8 was not lethal during the time the mice were observed (until P15) and pups were indistinguishable from Casp- $8^{\mathrm{WT}}$ littermates (Supplemental Figure 1, E-G). As Casp-8 is expressed in ECs of developing blood vessels in the retina (Supplemental Figure $1 \mathrm{H}$ ) and as this is a well-established model to postnatally study developmental angiogenesis (26), we used the growing retina for our purposes. Analysis of the vasculature (by staining with the endothelial marker isolectin B4 [IsoB4]) in P6 retinas showed that the total area covered by blood vessels and the vascular outgrowth were reduced in Casp- $8^{\mathrm{ECKO}}$ retinas compared with that in Casp$8^{\mathrm{WT}}$ littermates (Figure 1, B-D). The number of vessel branches was also decreased (Figure 1, E and F), indicating a reduced complexity of the vascular network in Casp- $8^{\mathrm{ECKO}}$ pups. In addition, we counted fewer sprouts at the angiogenic front in Casp- $8^{\mathrm{ECKO}}$ pups (Figure 1, G and H). Together, these data show that loss of Casp-8 in the endothelium results in vascular defects during developmental angiogenesis.

Necroptosis does not contribute to the vascular defects in Casp- $8^{\text {СКО }}$ pups. During postnatal angiogenesis in the retina, the vascular network develops to its final mature stage via a combination of different cellular processes, such as EC proliferation, migration, vessel maturation, and vessel remodeling. As Casp- 8 regulates cell death and survival and as this contributes to vessel remodeling and regression $(5,8,9)$, we checked to determine whether KO of Casp- 8 would result in EC death due to activation of the necroptotic pathway. In vivo, an indirect way to assess cell death is the quantification of regressing vessel branches, which leave collagen type IV $\left(\mathrm{ColIV}^{+}\right)$empty sleeves behind (27). Therefore, we analyzed vessel regression by quantifying the number of CollV ${ }^{+} \mathrm{IsoB}_{4}^{-}$ sleeves and found no differences between genotypes (Figure 2, A and $\mathrm{B}$ ). In line with these results, pericyte coverage analyzed by costaining of the pericyte marker desmin and IsoB4 revealed no differences between Casp- $8^{\mathrm{ECKO}}$ and Casp- $8^{\mathrm{WT}}$ retinas, indicating that vessel maturation and stabilization were also not affected (Figure 2, C and D). Interestingly, we noticed a small but significant decrease in the number of cleaved caspase- $3^{+}\left(\right.$cCasp- $\left.3^{+}\right)$and TUNEL $^{+}$ECs in Casp- $8^{\text {ECKO }}$ pups (Figure 2, E-H), suggesting that Casp-8-mediated apoptosis via the extrinsic cell death signaling pathway may have a small but significant contribution to the physiological remodeling process, without, however, affecting overall vessel regression. To further explore this hypothesis, we first analyzed the ability of ECs to die by necroptosis in vitro. For this purpose, we counted the number of propidium iodide-positive $\left(\mathrm{PI}^{+}\right)$ cells (PI detects apoptotic and necroptotic cells; ref. 28) in WT (Casp-8 ${ }^{\text {WT }}$ ) and Casp-8-knockdown (Casp- ${ }^{\mathrm{KD}}$ ) HUVECs using a lentivirus-mediated shRNA KD system (ref. 29 and Supplemental Figure 2A). Both Casp- $8^{\mathrm{WT}}$ and Casp $-8^{\mathrm{KD}}$ HUVECs showed a slight, 
A

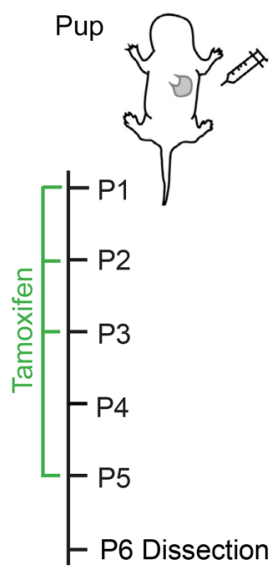

B
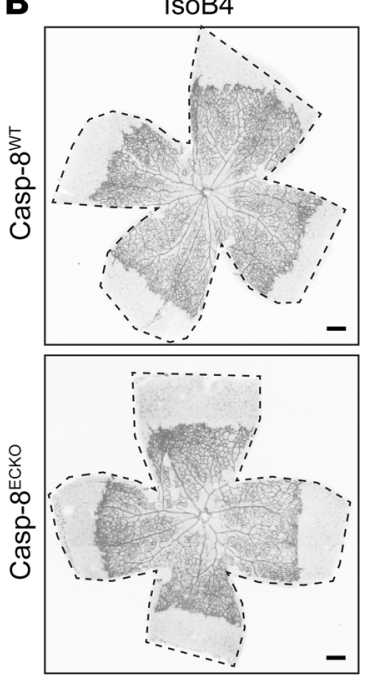

D

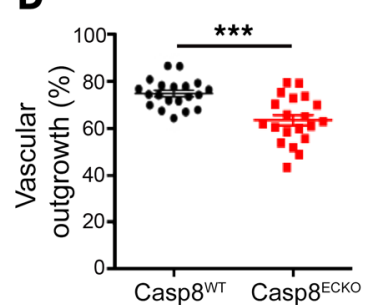

$\mathbf{E}$
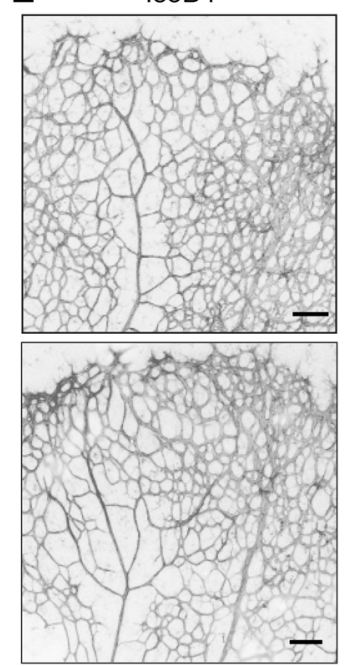

$\mathbf{F}$

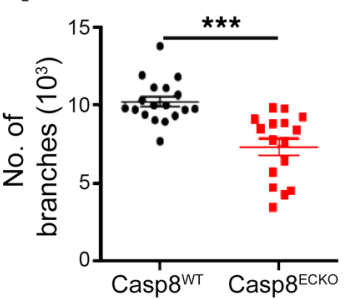

G $\quad \mid \mathrm{soB} 4$
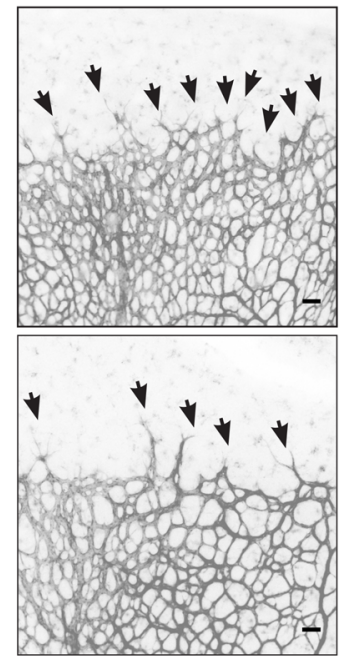

H

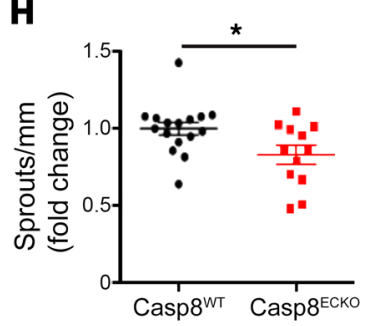

Figure 1. Postnatal EC-specific KO of Casp-8 results in impaired angiogenesis. (A) Schematic showing tamoxifen administration in pups. (B) Representative images of whole-mount P6 retinas stained with IsoB4 (ECs) in Casp-8 ${ }^{\text {WT }}$ and Casp- ${ }^{\text {ECKo }}$ mice. Dashed black lines highlight the total retina area. (C and D) Quantification of vessel area (C; $n=22$ WT, $n=21$ ECKO) and retina vessel outgrowth (D; $n=20$ WT, $n=20$ ECKO). (E) Representative higher magnifications of the retina stained with IsoB4. (F) Quantification of number of branches ( $n=18 \mathrm{WT}, n=16$ ECKO). (G) Representative images of the retina angiogenic front stained with IsoB4. Black arrows point to EC sprouts. (H) Quantification of the number of sprouts per front length showing reduced number of sprouts in Casp- $8{ }^{\text {ECKO }}$ retinas ( $n=16$ WT, $n=12$ ECKO). For C, D, F, and $\mathbf{H}$, data are shown as mean \pm SEM from 4 independent litters. ${ }^{*} P<0.05 ;{ }^{* *} P<$ 0.001, 2-tailed unpaired Student's $t$ test. Scale bars: $100 \mu \mathrm{m}$ (B); $50 \mu \mathrm{m}$ (E); $20 \mu \mathrm{m}$ (G).

though not significant, increase in the number of $\mathrm{PI}^{+}$cells upon TRAIL or TNF stimulation (Supplemental Figure 2B). As a positive control to demonstrate that HUVECs were not just resistant to cell death, we knocked down c-FLIP (c-FLIP ${ }^{\mathrm{KD}}$ ), the intrinsic inhibitor of Casp-8. c-FLIP ${ }^{\mathrm{KD}}$ ECs presented a significant increase in cell death upon TNF or TRAIL stimulation (Supplemental Figure 2B). As vessel development in the retina is regulated by hypoxia (30) and hypoxia can be a modulator of cell death (31), we also performed the same experiments under hypoxic conditions, which produced the same results (Supplemental Figure 2C).

Finally, to determine whether necroptosis could contribute to the observed phenotype in vivo, we genetically deleted MLKL (the ultimate effector of necroptosis; ref. 13) in the Casp- ${ }^{\text {ЕСКО }}$ mice by crossing Cdh5-(PAC)-CreERT $2 \times$ Casp- $8^{\mathrm{fl} / \mathrm{fl}}$ mice with a MLKL ${ }^{\mathrm{KO}}$ mouse line. The vessel area and the number of vessel branches at P6 were not affected in heterozygous or homozygous MLKL ${ }^{\mathrm{KO}}$ pups compared with WT littermates (Supplemental Figure 3, A-D), indicating that MLKL alone did not contribute to angiogenesis. However, the vessel area and the number of vessel branches as well as the sprouts at the angiogenic front were still reduced in Casp- $8^{\mathrm{ECKO}} / \mathrm{MLKL}^{\mathrm{KO}}$ pups (compared with Casp-8 ${ }^{\mathrm{WT}} / \mathrm{MLKL}^{\mathrm{KO}}$ pups) (Figure 2, I-M), show- ing that blocking necroptosis in Casp- $8^{\mathrm{ECKO}}$ pups did not rescue the vascular defects.

Our data indicate that the loss of Casp-8 in ECs during postnatal development does not induce cell death via necroptosis. Even though we found a mild decrease in the number of cCasp $-3^{+}$and TUNEL ${ }^{+}$ECs at P6 in Casp- ${ }^{\text {ECKO }}$ pups, this did not affect the percentage of vessel regression overall, indicating that extrinsic cell death is not an active driver of vessel pruning during postnatal vascular development. Our data further suggest that Casp-8 regulates developmental angiogenesis in a cell death-independent way.

Casp -8 regulates VEGF-induced EC sprouting, proliferation, and migration. It has been shown that Casp-8 has diverse cell deathindependent functions, for example, in the regulation of cell migration $(14,32)$. Thus, to further characterize the EC phenotype and to understand which processes are impaired upon loss of Casp-8, we analyzed the response of Casp- $8^{\mathrm{KD}}$ ECs upon VEGF stimulation. To do so, we analyzed EC sprouting in vitro using the bead sprouting assay (33). While KD of Casp-8 (using a specific siRNA, Supplemental Figure 4A) had no effect on unstimulated conditions, Casp- $8^{\mathrm{KD}}$ ECs failed to respond to VEGF stimulation (Figure 3, A and B). Additionally, we tested the response of Casp- $8^{\mathrm{KD}}$ ECs to VEGF stimulation using the tube-formation assay. 
A
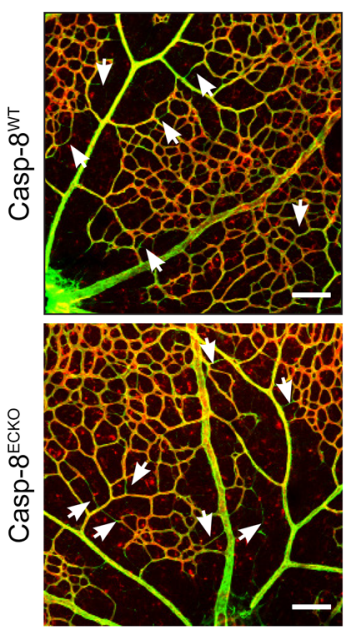

B
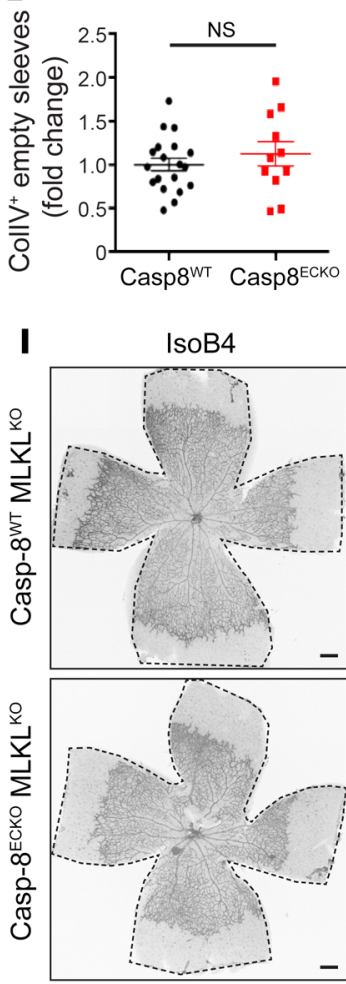
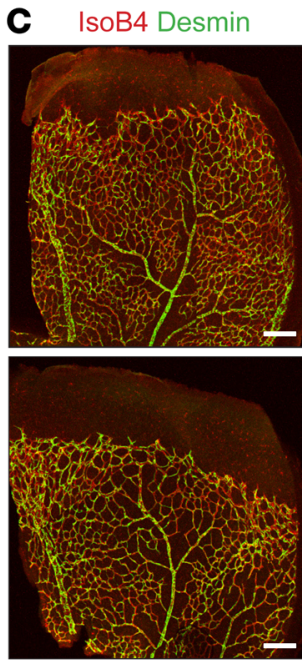

D
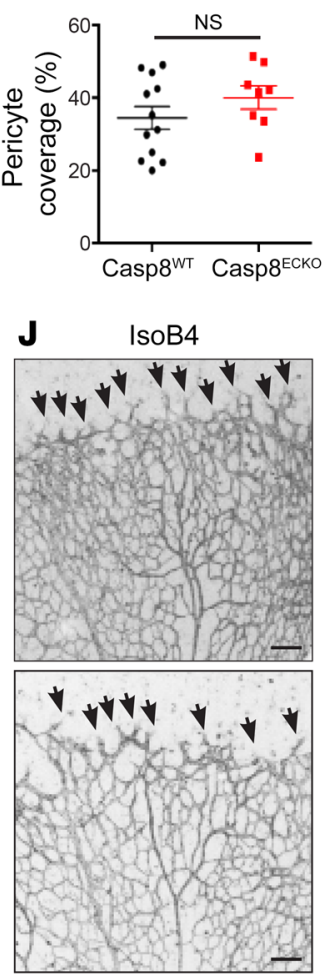
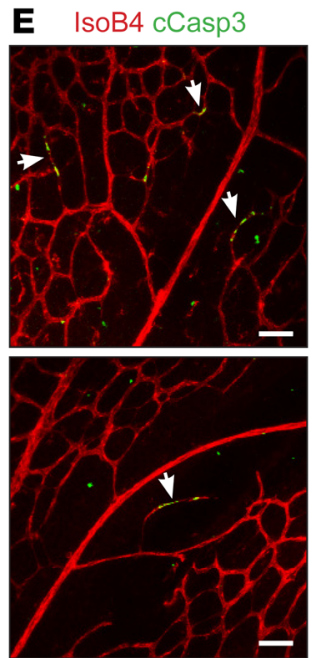

$\mathbf{F}$
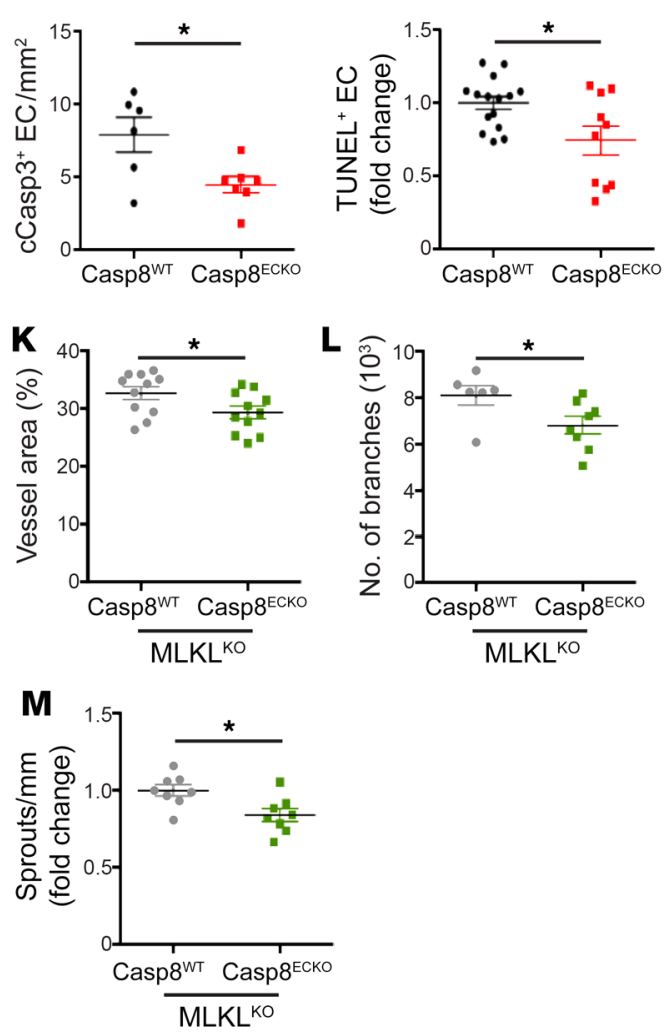

Figure 2. Loss of Casp-8 in ECs does not result in vessel regression or necroptosis. (A) Retinas from P6 pups were costained with IsoB4 and collagen IV (CollV) in Casp-8 $8^{\text {WT }}$ and Casp-8 ${ }^{\mathrm{ECKO}}$ retinas. White arrows point to CollV ${ }^{+}$IsoB4- empty sleeves. (B) Quantification of relative vessel regression shows no significant differences between genotypes ( $n=19 \mathrm{WT}, n=11 \mathrm{ECKO}$ ). (C) Representative images of pericyte coverage. Retinas were costained with desmin (pericyte marker) and IsoB4. (D) Quantification of Desmin ${ }^{+}$area per vascular area (\%) shows no significant differences between genotypes ( $n=12$ WT, $n=8$ ECKO). (E) Representative images of apoptotic ECs (white arrows), costained with IsoB4 and cCasp-3. (F) Quantification of cCasp- $3^{+}$IsoB4+ cells per vessel area revealing fewer apoptotic ECs in Casp- $8^{\mathrm{ECKO}}$ retinas compared with Casp- $8^{\mathrm{WT}}$ ( $n=6 \mathrm{WT}, n=7$ ECKO). (G) Representative images of retinas costained with IsoB4 and TUNEL (black arrows point to TUNEL+ECs). Images showing IsoB4 were transformed to gray colors with ImageJ for better visualization. (H) Quantification of relative amount of TUNEL+ECs per vessel area also shows decreased numbers of apoptotic ECs in Casp- 8 ECKO retinas ( $n=15$ WT, $n$ $=10$ ECKO). (I and J) Representative images of the retinal vasculature stained with IsoB4 in Casp-8 ${ }^{\mathrm{WT}} / \mathrm{MLKL}^{\mathrm{KO}}$ and Casp- $8^{\mathrm{ECKO}} / \mathrm{MLKL}^{\mathrm{KO}}$ mice. Black arrows point to EC sprouts. (K-M) Quantitative analysis showing reduced vessel area (K; $n=11 \mathrm{WT}, n=11 \mathrm{ECKO}$ ), number of branches (L; $n=6 \mathrm{WT}, n=8 \mathrm{ECKO})$, and reduced number of sprouts per front area ( $\mathbf{M} ; n=6$ WT, $n=8$ ECKO) in Casp- $8^{\mathrm{ECKO}} / \mathrm{MLKL}^{\mathrm{KO}}$ retinas compared with Casp-8 ${ }^{\mathrm{WT}} / \mathrm{MLKL}^{\mathrm{KO}}$ littermates. For $\mathbf{B}, \mathbf{D}, \mathbf{F}$, $\mathbf{H}$, and $\mathbf{K}-\mathbf{M}$, data are shown as mean \pm SEM from at least 3 independent litters. ${ }^{*} P<0.05$, 2-tailed unpaired Student's $t$ test. Scale bars: $100 \mu \mathrm{m}$ (A, C, and I); $20 \mu \mathrm{m}$ (E and $\mathbf{G}) ; 50 \mu \mathrm{m}(\mathrm{J})$. 

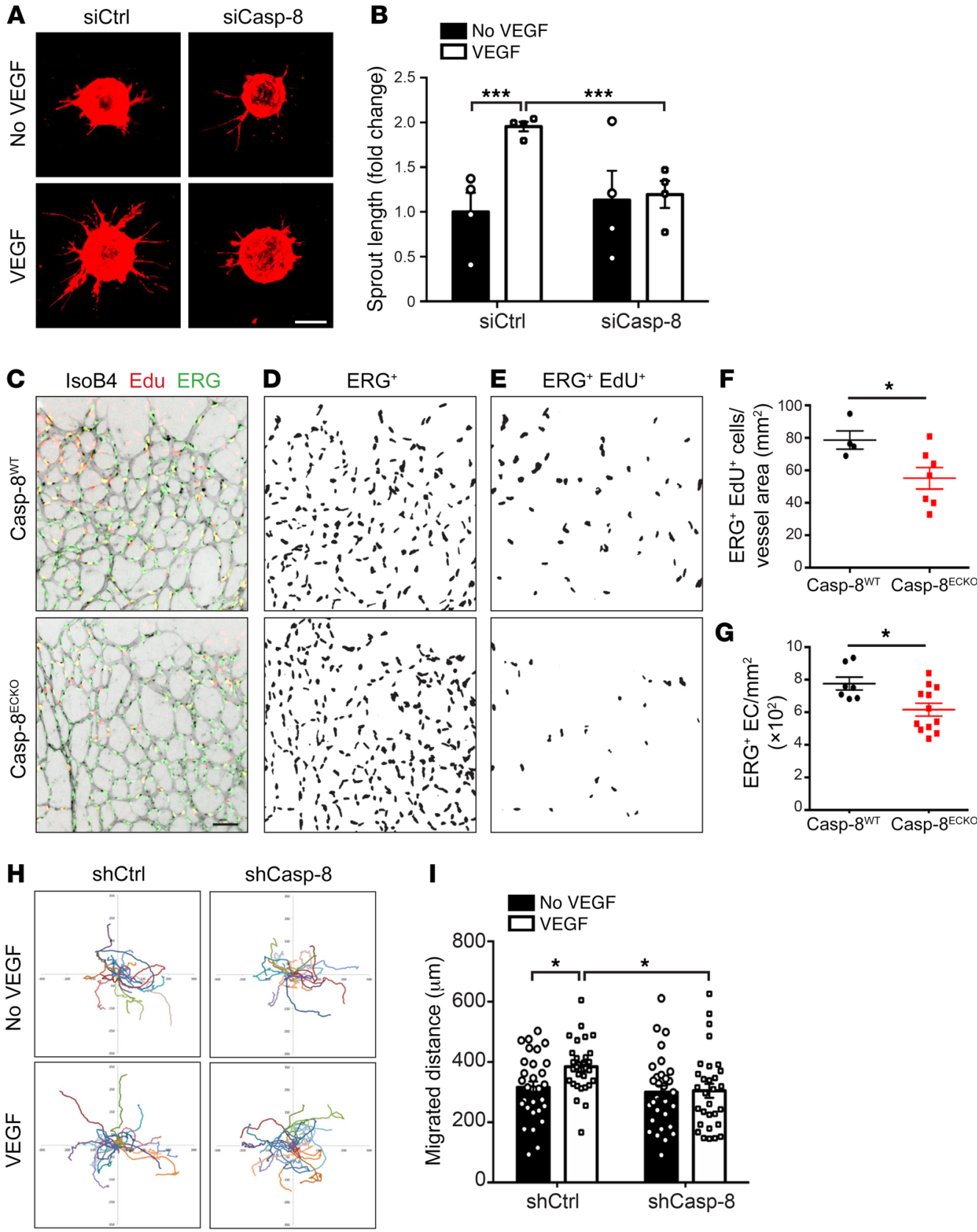

Figure 3. Loss of Casp-8 in ECs impairs sprouting, proliferation, and migration. (A) Representative images of the bead-sprouting assay using HUVECs transfected with control siRNA (siCtrl) or Casp-8 siRNA (siCasp-8) and treated with VEGF ( $50 \mathrm{ng} / \mathrm{ml}$ ) for 24 hours. (B) Quantitative analysis of total sprout length showing that VEGF is not able to induce vessel sprouting in the absence of Casp-8. Approximately 20 beads per condition were quantified. $n=4$. (C) Representative images of the retinal vasculature costained with IsoB4, EdU (labels proliferating cells), and ERG (labels EC nuclei) in Casp-8WT and Casp- $8^{\mathrm{ECKO}}$ mice. IsoB4 single channel was transformed to gray colors and inverted with Imagej for better visualization. ( $D$ and E) Masks obtained by Imagej of ERG ${ }^{+}$cells (D) and of ERG ${ }^{+}$EdU ${ }^{+}$proliferating ECs (E). (F and G) Quantification of total number of ECs per retina area (F; $n=7 \mathrm{WT}, n=12$ ECKO) and proliferating ECs per vessel area (G; $n=4$ WT, $n=7 \mathrm{ECKO}$ ), revealing lower absolute EC numbers and fewer proliferating ECs in Casp- $8^{\text {ECKO }}$ retinas compared with Casp- $8^{\text {WT }}$ littermates. Data from 2 independent litters. (H) Single-cell motility tracks of HUVECs infected with a control (shCtrl) or Casp-8 shRNA lentivirus (shCasp-8) and treated with VEGF (50 ng/ml) for 12 hours. Migration origin of each cell was overlaid at the zero-crossing point. (I) Quantification of the total migration distance of HUVECs in H showing that VEGF-induced migration was impaired in Casp- $8^{\mathrm{KD}} \mathrm{ECs}$. At least 30 cells per condition were quantified. $n=3$. For $\mathbf{B}, \mathbf{F}, \mathbf{G}$, and $\mathbf{I}$, data are shown as mean $\pm \mathrm{SEM}$. ${ }^{*} P<0.05$; ${ }^{* * *} P<0.001$, 2-way ANOVA with Bonferroni's multiple comparisons test (B and I); ${ }^{*}<<0.05$, 2-tailed unpaired Student's $t$ test (F and G). Scale bars: $200 \mu \mathrm{m}$ (A); $50 \mu \mathrm{m}$ (C).

Consistently, the ability of Casp- $8^{\mathrm{KD}}$ ECs to form capillary-like tube structures upon VEGF stimulation was also significantly attenuated (Supplemental Figure 4, B and C).

We next determined whether Casp-8 activity was required for the proper response of ECs to VEGF stimulation. Using a lumines- cent Glo assay, we first confirmed that ECs have a basal Casp-8 activity, which could be blocked with the Casp-8 inhibitor Z-IETDFMK (ZIETD, Supplemental Figure 4D). As a positive control for our assay, we treated ECs with a combination of cycloheximide (CHX) and TNF to induce apoptosis, which strongly increased 
A
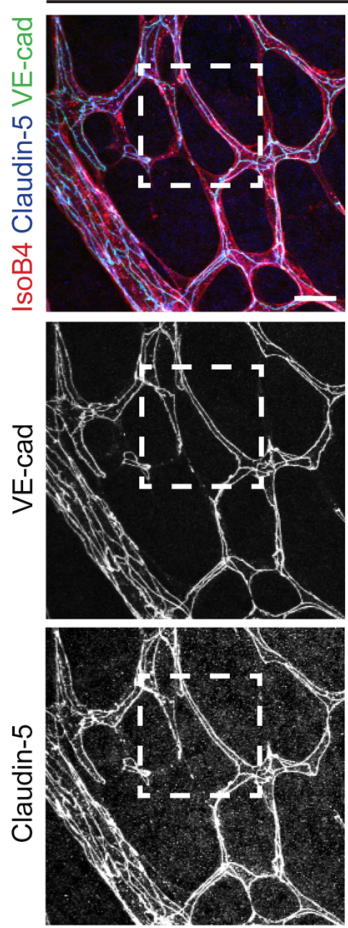

Casp-8wT
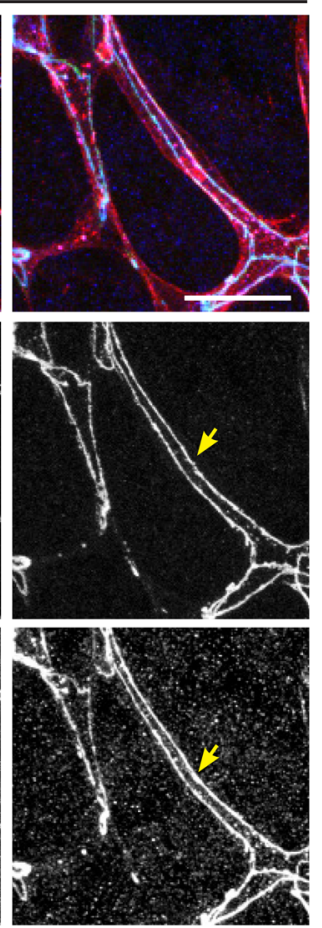

Casp-8 $8^{\mathrm{ECKO}}$
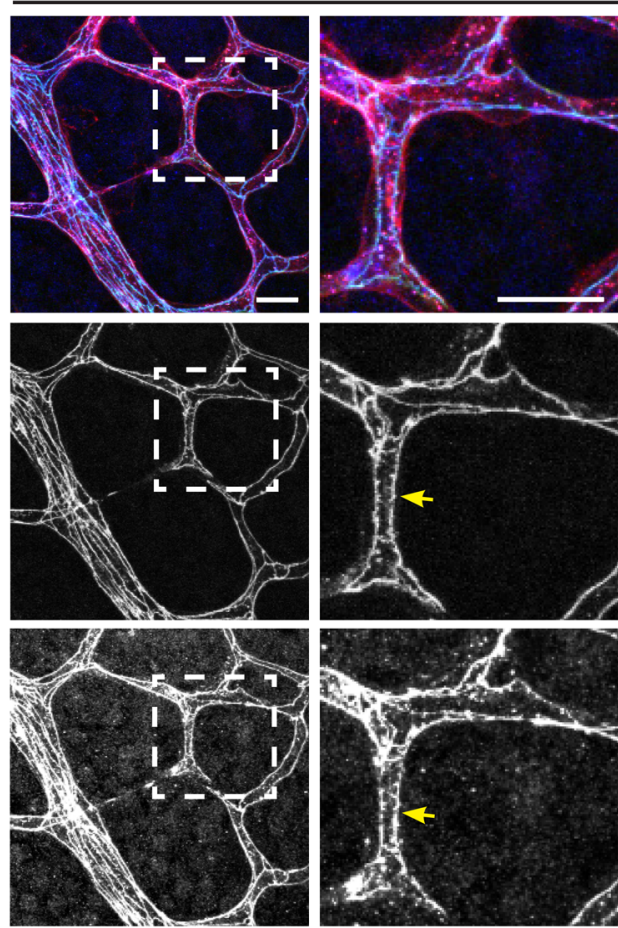

C

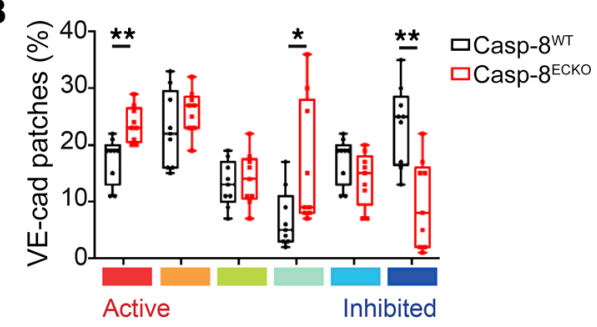

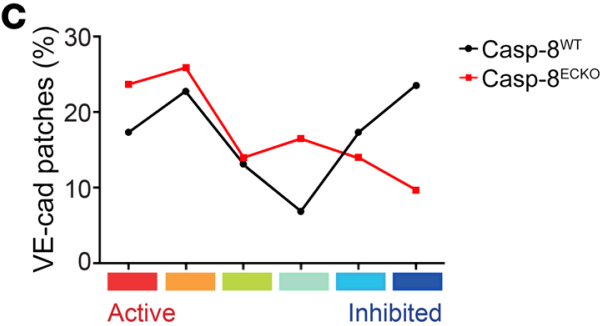

Figure 4. Casp-8 is necessary for maintaining EC junction stability at the retina plexus in vivo. (A) Representative images of Casp- $8^{\text {WT }}$ and Casp- $8^{\text {ECKO }}$ retinas stained with IsoB4, VE-cadherin, and claudin-5 (insets of left panels are shown on the adjacent right panels), showing that junctions are more serrated and discontinuous at the retina plexus in Casp- 8 ЕсKo compared with Casp-8 ${ }^{\text {WT }}$ mice (yellow arrows). Images of VE-cadherin and Claudin- 5 single channels were transformed to gray colors with Image for better visualization. (B) Quantification of the percentage of VE-cadherin patches showing a significant increase in the number of highly active VE-cadherin patches and a lower number of highly inhibited patches in Casp- $8^{\text {ECKO }}$ compared with Casp-8 ${ }^{\text {WT }}$ mice. Each box shows the median percentage of patches of that type (line) and upper and lower quartiles (box). The whiskers extend to the most extreme data within 1.5 times the interquartile range of the box. ${ }^{*} P<0.05$; ${ }^{* *} P<$ 0.01 , Dirichlet regression model with 2-tailed Mann-Whitney $U$ test for each state. $n=9 \mathrm{WT}, n=9$ ECKO. (C) Average of the differential distribution of the percentage of VE-cadherin patches in Casp- $8^{\mathrm{WT}}$ and Casp-8 8 Еко retinas. Scale bars: $20 \mu \mathrm{m}$ (A).
Casp-8 activity, as expected (Supplemental Figure 4D). Functionally, blocking Casp-8 activity in ECs resulted in the same defects as the KD of Casp-8, as ZIETD-treated ECs did not form sprouts upon VEGF stimulation (Supplemental Figure 4, E and F), indicating that Casp-8 activity was required for proper angiogenesis.

To assess whether changes in EC proliferation could contribute to the reduced vascular area in Casp $-8^{\mathrm{ECKO}}$ retinas, we performed proliferation experiments. shRNA-mediated Casp- 8 KD in HUVECs resulted in reduced proliferation upon stimulation with VEGF or FGF, as determined by BrdU incorporation (Supplemental Figure 4G). Also, siRNA-transfected Casp- $8^{\mathrm{KD}}$ HUVECs had a reduced response to VEGF stimulation in the WST-1 cell proliferation and viability assay (Supplemental Figure $4 \mathrm{H}$ ). In vivo, the total number of ECs, as determined by counting $\mathrm{ERG}^{+}$nuclei per retina area (ERG is an EC-specific transcription factor; ref. 34) was reduced (Figure 3, C, D, and F). EdU labeling of proliferating cells in $\mathrm{P} 6$ retinas showed significantly fewer $\mathrm{EdU}^{+} \mathrm{ERG}^{+}$cells per vessel area in Casp- $8^{\mathrm{ECKO}}$ pups compared with Casp- $8^{\text {WT }}$ littermates (Figure 3, C, E, and G), indicating that reduced EC proliferation could account for the reduced vessel area.

EC migration is also required for proper expansion of the vascular network. We therefore checked to determine whether
KD of Casp-8 in ECs could also affect this process. In a classical scratch-wound assay, VEGF stimulation of Casp- $8^{\text {WT }}$ ECs led to almost $80 \%$ closure of the wound, whereas KD of Casp-8 reduced VEGF-induced wound closure (Supplemental Figure 4, I and J). To determine whether Casp-8 affected migration independently of its effects on proliferation, we performed live-imaging experiments and tracked the movement of nondividing single Casp- $8^{\mathrm{KD}}$ ECs. Indeed, the total migration distance of ECs after 12 hours of VEGF stimulation was reduced when Casp-8 was knocked down (Figure 3, H and I). Together, these results show that Casp8 regulates EC proliferation and migration, which contributes to VEGF-induced sprouting.

Loss of Casp-8 affects the organization of adherens and tight junctions in ECs. Aside from being important for vascular homeostasis and vessel stability, dynamic turnover of vascular endothelial cadherin (VE-cadherin) is crucial for proper vessel sprouting and elongation during angiogenesis (35-39).

As both sprouting and migration were impaired in Casp- $8^{\text {Еско }}$ mice and as Casp- 8 has been shown to regulate the stability of cell junctions in the epidermis (40), we analyzed the distribution of VE-cadherin in vivo in the sprouting front and in the plexus of the growing vasculature of $\mathrm{P} 6$ retinas. By using an established image software analysis and classification key $(35,41)$, we distinguished 
A

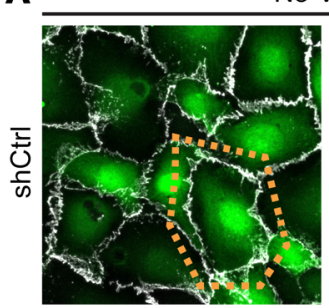

No VEGF

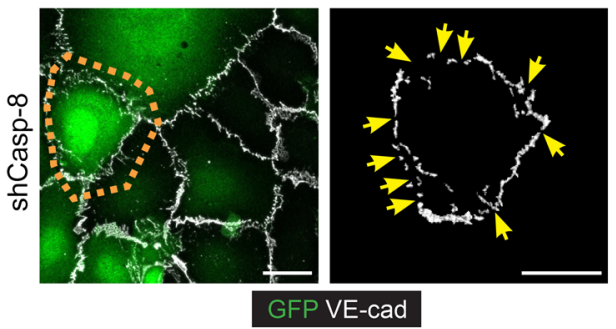

C

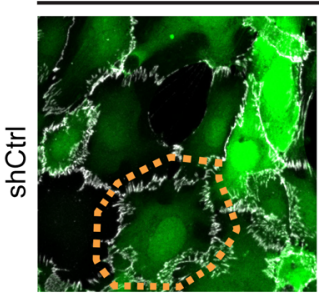

$60 \mathrm{~min}$ VEGF
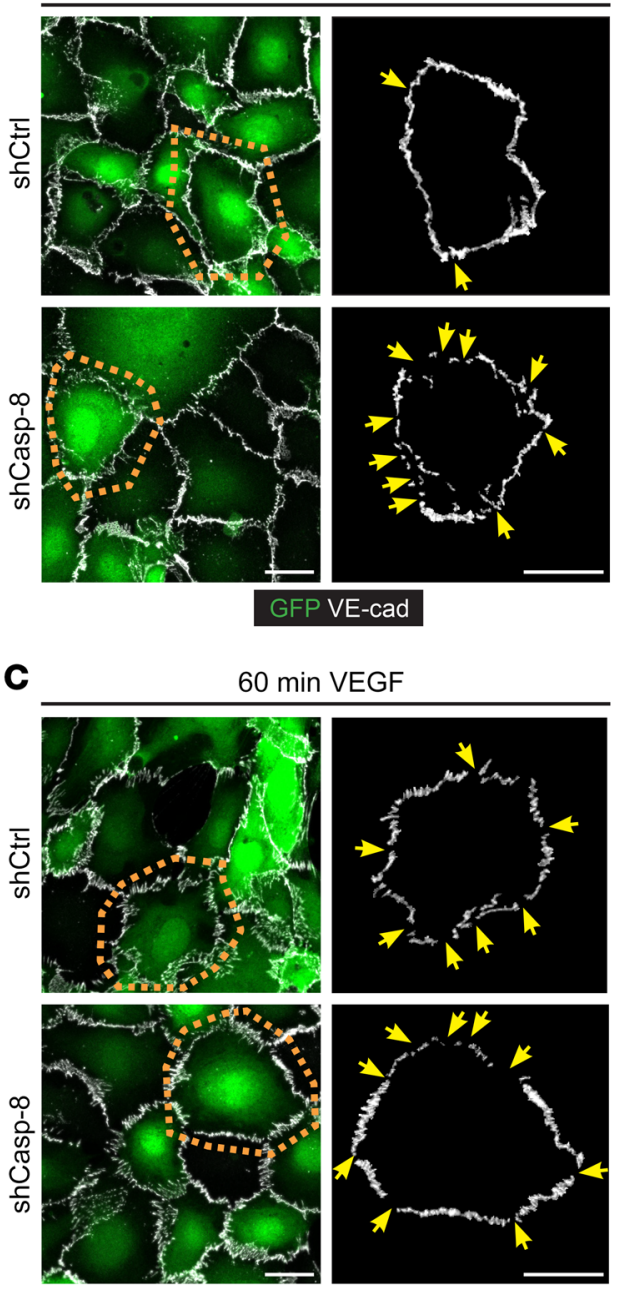

B
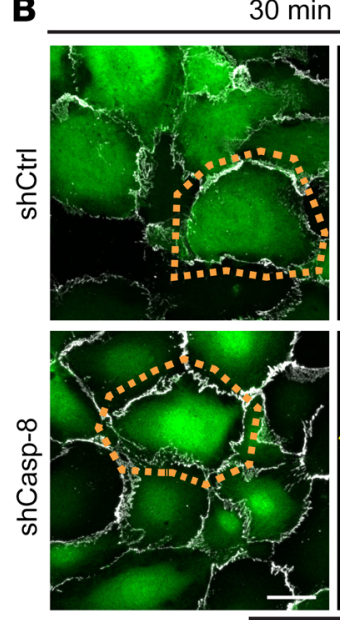

GFP VE-cad

D

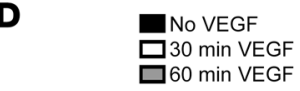

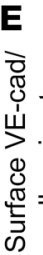

$30 \mathrm{~min}$ VEGF

-cad
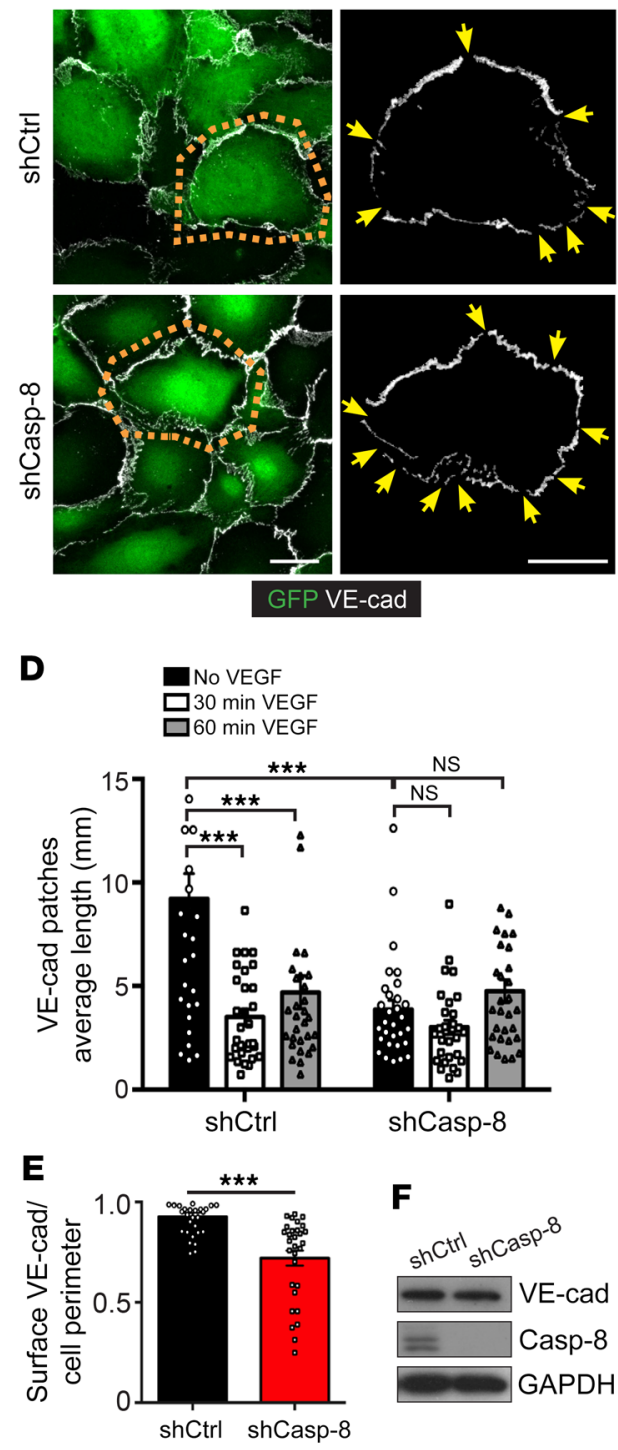

Figure 5. VE-cadherin distribution in ECs is affected in the absence of Casp-8 in vitro. (A-C) Representative images of VE-cadherin (VE-cad) staining in confluent HUVECs infected with shCtrl or shCasp-8 lentivirus (GFP+) with (B and C) or without (A) VEGF (50 ng/ml) stimulation. Results of tracing VE-cadherin staining of the single cells within orange dotted insets is shown in the adjacent right panels. Yellow arrows point to empty VE-cadherin spots. (D) Quantification of the average length of VE-cadherin patches showing that VEGF-induced VE-cadherin reorganization is impaired in Casp- $8^{\mathrm{KD}} \mathrm{ECs} .{ }^{* * *} P<0.001$, 2-way ANOVA with Bonferroni's multiple comparisons test. (E) Quantification of the total amount of VE-cadherin per cell perimeter showing less VE-cadherin in Casp- $8^{\mathrm{KD}} \mathrm{ECs}$. ${ }^{* * *} P<0.001$, 2-tailed unpaired Student's $t$ test. (F) Western blot showing unchanged total VE-cadherin protein levels in HUVECs infected with shCtrl or shCasp-8. For $\mathbf{D}$ and $\mathbf{E}$, at least 15 cells per condition were quantified. $n=3$. Data are shown as mean \pm SEM. Scale bars: $20 \mu \mathrm{m}(\mathbf{A}-\mathbf{C})$. between remodeling (active) and stable (inhibited) VE-cadherin patches. As expected, Casp- $8^{\mathrm{WT}}$ retinas presented a highly active VE-cadherin pattern in the sprouting front that was only slightly affected in Casp- $8^{\text {ECKO }}$ pups (Supplemental Figure 5, A-C). More strikingly, Casp- $8^{\mathrm{E} \text { Со }}$ retinas had destabilized, more discontinuous (active) VE-cadherin staining in the back (plexus) of the retina, whereas Casp- $8^{\mathrm{WT}}$ retinas showed continuous (inhibited) patches of VE-cadherin (Figure 4, A-C). Analysis of the distribution of claudin- 5 in the plexus, one of the main transmembrane proteins found in tight junctions, showed the same discontinuous pattern in the back of Casp- $8^{\mathrm{ECKO}}$ retinas (Figure $4 \mathrm{~A}$ ).

Next, we confirmed the VE-cadherin phenotype in vitro. Consistent with previous studies (42), confluent control shRNAtransfected ECs (Casp-8 ${ }^{\text {WT }}$ ECs) showed a continuous (inhibited) VE-cadherin staining along the cell junctions (Figure 5A), which became serrated with a rope-ladder pattern (active) upon 30 or 60 minutes of VEGF stimulation (Figure 5, B and C), as analyzed by measuring the average length of individual VE-cadherin patches (Figure 5D). In contrast, the distribution of VE-cadherin in confluent
Casp- $8^{\mathrm{KD}}$ ECs was already discontinuous/serrated in unstimulated conditions, and VE-cadherin did not further rearrange upon VEGF stimulation (Figure 5, A-D). Moreover, VE-cadherin levels at the cell perimeter were reduced under basal conditions (Figure $5 \mathrm{E})$. However, total VE-cadherin protein levels were unchanged (Figure 5F), indicating that VE-cadherin was not degraded, but only mislocalized. Taken together, our data show that the absence of Casp-8 in ECs affects the proper formation of adherens and tight junctions in the postnatal retina as well as the VEGF-induced remodeling of VE-cadherin in vitro.

Loss of Casp- 8 results in the basal activation of the p 38 MAPK. We next pursued experiments aimed at identifying the signaling pathways involved in EC proliferation, sprouting, and migration that could be altered in Casp- $8^{\mathrm{KD}}$ ECs. In the absence of Casp-8, phosphorylation of Akt, ERK, and FAK was not affected under basal conditions or upon VEGF stimulation (Supplemental Figure 6, A-D). However, knocking down Casp-8 or blocking its activity resulted in increased basal p38 MAPK phosphorylation (Figure 6, A-C). Still, Casp- $8^{\mathrm{KD}}$ ECs or HUVECs treated with ZIETD were 
A

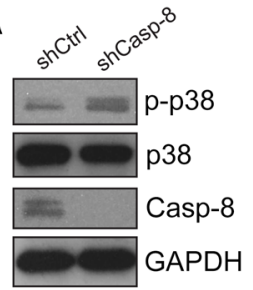

B

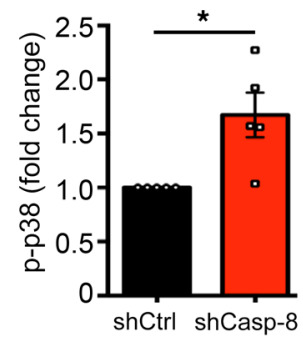

$\mathbf{C}$

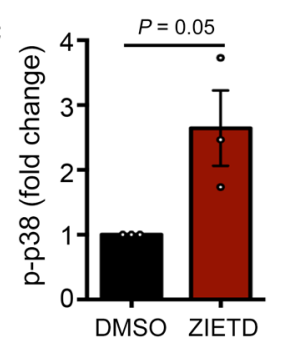

D

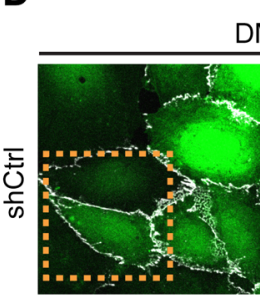

DMSO

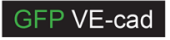
SB203580
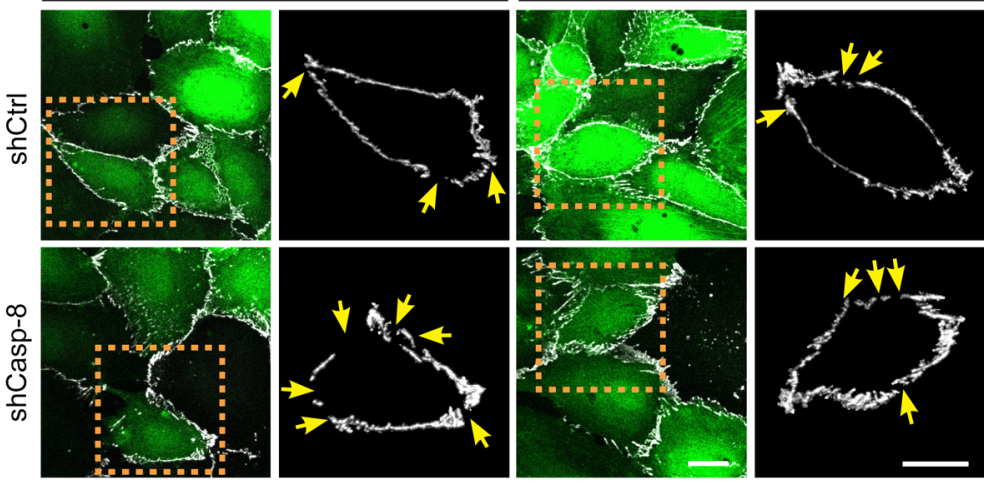

E
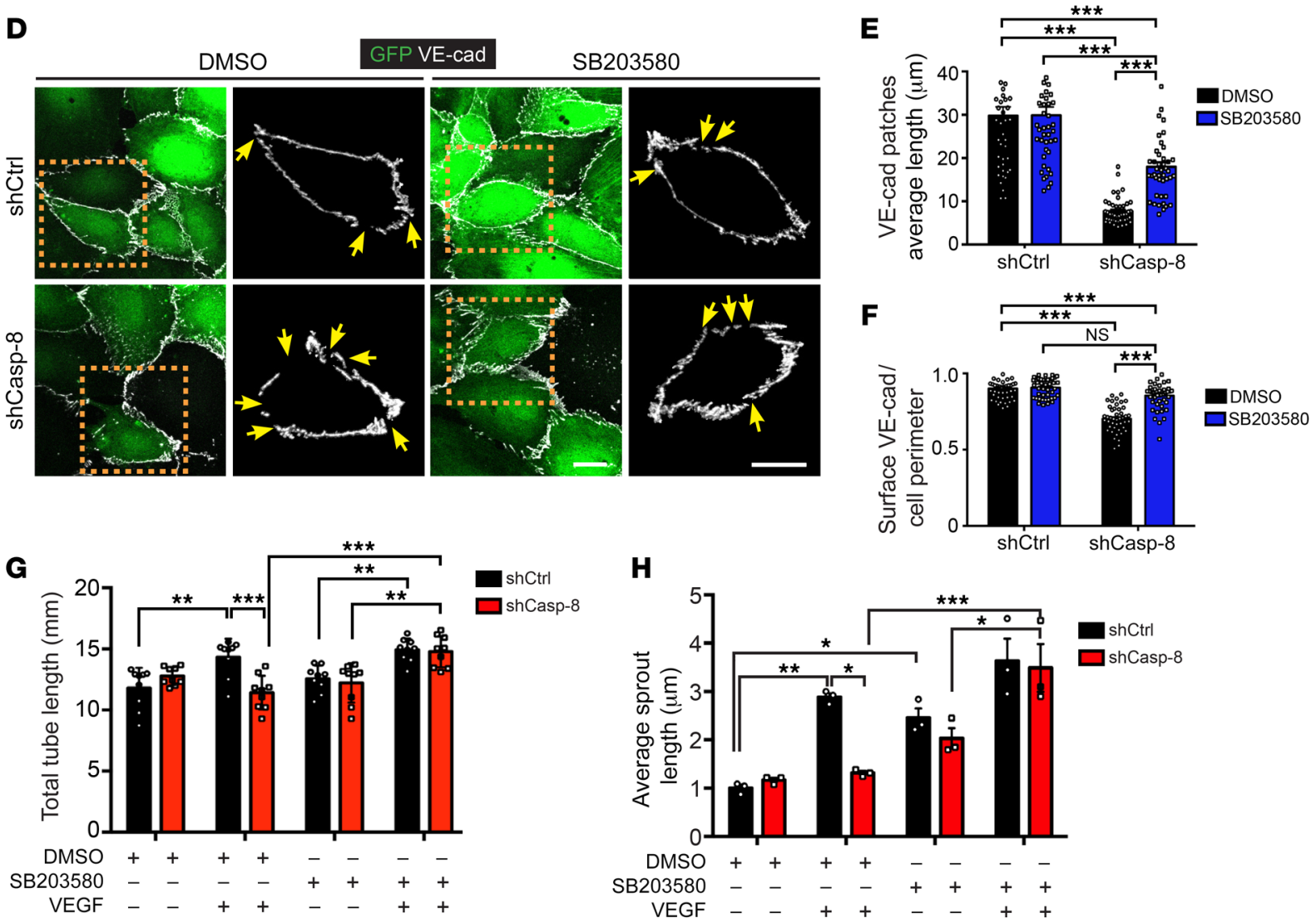

Figure 6. Loss of Casp-8 results in basal activation of p38 MAPK, VE-cadherin instability, and defects in angiogenesis in vitro. (A)Western blots showing increased p-p38 under basal conditions in Casp-8 ${ }^{\mathrm{KD}}$ (shCasp-8) ECs compared with control. (B) Quantification of p-p38 as in A. $n=5$. (C) Quantification of p-p38 of HUVECs treated with ZIETD ( $10 \mu \mathrm{M}, 16$ hours) under basal conditions, showing that blocking Casp-8 activity also induces increased basal p-p38. $n$ = 3. (D) Images of VE-cadherin staining in shCtrl and shCasp-8-infected HUVECs treated with or without p38 inhibitor (SB203580, $1 \mu \mathrm{M}, 16$ hours). Yellow arrows point to empty VE-cadherin spots. (E) Quantification of VE-cadherin average patch length from cells as in $\mathbf{H}$. (F) Quantification of the total amount of VE-cadherin per cell perimeter reveals that inhibition of $\mathrm{p} 38$ (SB203580) in Casp-8 ${ }^{\mathrm{KD}} \mathrm{ECs}$ restores VE-cadherin to control levels. At least 15 cells per condition were quantified. $n=3$. (C) Quantification of total tube length of HUVECs treated as in E showing that blocking p38 in Casp- $8^{\mathrm{KD}}$ ECs restores VECF-induced tube formation. 3 fields per condition were quantified. $n=4$. (H) Quantitative analysis of total sprout length showing that inhibition of p38 rescues VEGFinduced EC sprouting in Casp- $8^{\mathrm{KD}} \mathrm{ECs}$. Approximately 20 beads per condition were quantified. $n=3$. For $\mathbf{B}$, C, and $\mathbf{E}-\mathbf{H}$, data represent mean $\pm S E M$. ${ }^{*} P<$ $0.05,1$-sample $t$ test $(\mathbf{B}$ and $\mathbf{C}) ;{ }^{*} P<0.05 ;{ }^{*} P<0.01 ;{ }^{* *} P<0.001$, 2-way ANOVA with Bonferroni's multiple comparisons test (E-H). Scale bars: $20 \mu \mathrm{m}(\mathbf{D})$.

able to further activate p38 upon VEGF stimulation (Supplemental Figure 6, A and E-G). As increased activation of $\mathrm{p} 38$ has been linked to the destabilization of VE-cadherin in the endothelium $(43,44)$, and as VE-cadherin distribution was already altered in basal conditions in Casp- ${ }^{\mathrm{KD}}$ ECs (Figure 5, A and D), we explored whether the increased basal levels of phosphorylated p38 (p-p38) in Casp- $8^{\mathrm{KD}} \mathrm{EC}$ s were linked to the changes in the distribution of VE-cadherin. For this, we blocked p38 activity in confluent ECs with the specific p38 inhibitor SB203580 (45). Even though the length of individual surface VE-cadherin patches was only partially rescued (Figure 6, D and E), the total amount of VE-cadherin at the cell surface of Casp- ${ }^{\mathrm{KD}}$ ECs was fully recovered (Figure 6F), indicating that indeed the basal activation of $\mathrm{p} 38$ was linked to VEcadherin localization.

To determine whether the basal increase in p-p38 in Casp- $8^{\mathrm{KD}}$ ECs was also sufficient to functionally block VEGF-induced angiogenesis in Casp- $8^{\mathrm{KD}}$ ECs, we performed the tube-formation and bead-sprouting assays in the presence of the p38 inhibitor. Indeed, inhibition of $\mathrm{p} 38$ rescued the ability of Casp- ${ }^{\mathrm{KD}}$ ECs to respond to VEGF and to form both tubes (Figure 6G and Supplemental Figure $6 \mathrm{H}$ ) and sprouts (Figure $6 \mathrm{H}$ ) of a length similar to that of Casp-8 ${ }^{\mathrm{WT}}$ ECs. 
A

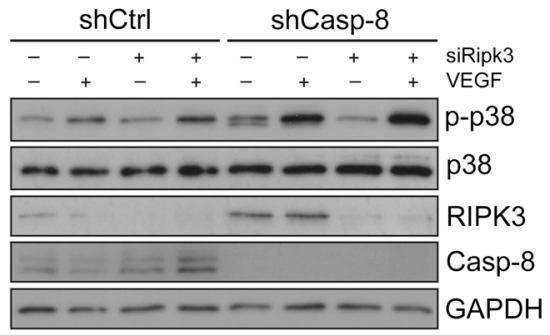

$\mathbf{E}$

E $\mathbf{s h C t h}_{\text {shct }}$

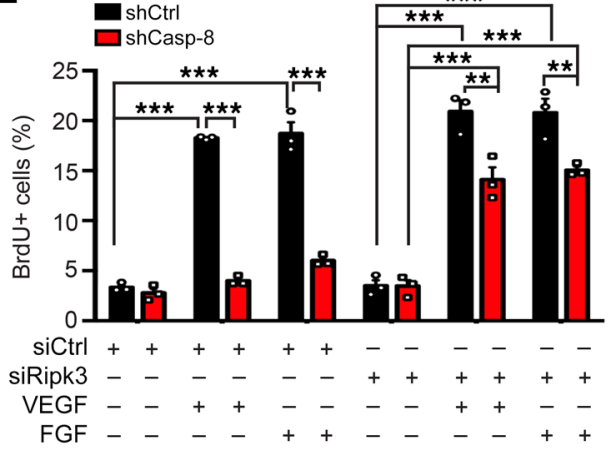

H

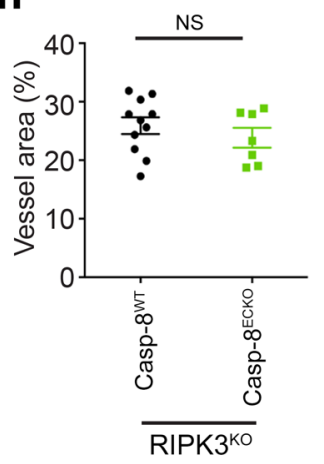

I

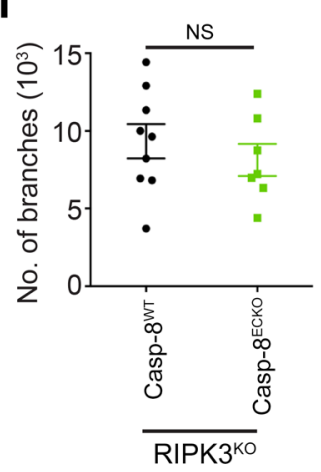

B

口shCtrl

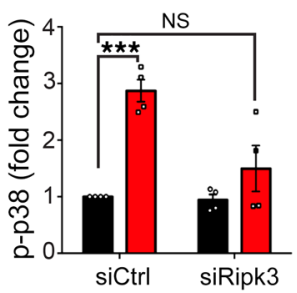

C

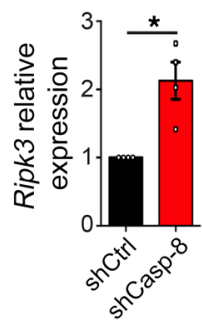

D

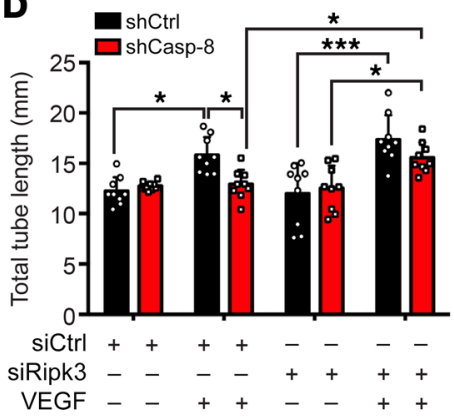

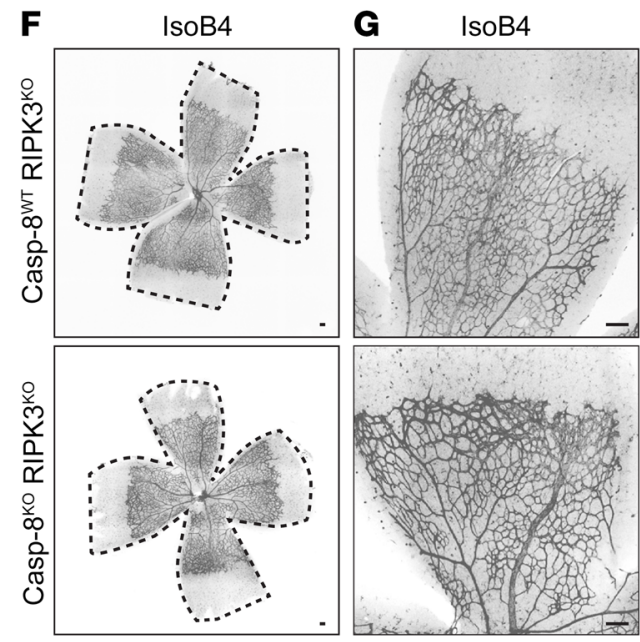

J

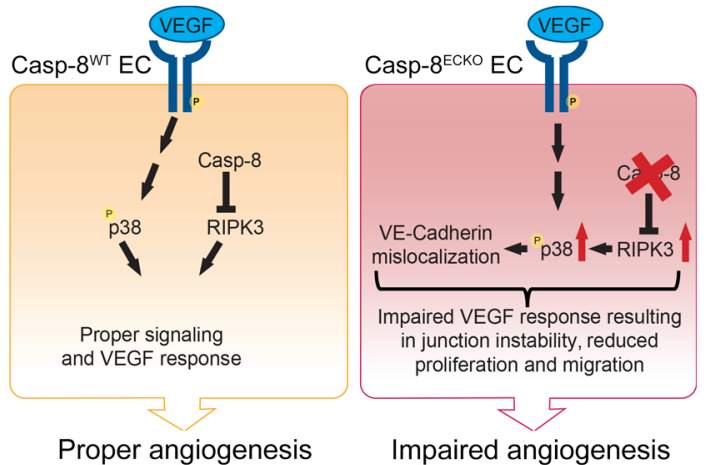

Figure 7. RIPK3 acts downstream of Casp-8 to regulate angiogenesis. (A) Western blot showing that knocking down RIPK3 in Casp- ${ }^{\mathrm{KD}}$ ECs rescues the basal hyperphosphorylation of p38. Notice that shCasp-8 HUVECs have increased RIPK3 protein levels. (B) Quantification of Western blots under basal conditions as in $\mathbf{A}$. $n=4$. (C) Quantitative PCR (qPCR) of shCtrl or shCasp-8-infected HUVECs showing increased mRNA expression of Ripk3 in the absence of Casp-8. $n=4$. (D) Quantification of total tube length of shCtrl or shCasp-8-infected HUVECs that were cotransfected with control siRNA or Ripk3 siRNA and treated with or without VEGF $\left(50 \mathrm{ng} / \mathrm{mL}\right.$ ) for 4 hours. Ten fields per condition were quantified. $n=3$. (E) BrdU+ cells were quantified in control and Casp- ${ }^{\mathrm{KD}}$ ECs transfected with Ripk3 siRNA and with or without VEGF $(50 \mathrm{ng} / \mathrm{mL})$ or FGF $(50 \mathrm{ng} / \mathrm{mL})$ stimulation for 24 hours. Around 50 cells per condition were quantified. $n=3$. (F and $\mathbf{G}$ ) Representative images of the retinal vasculature stained with IsoB4 in Casp- $8^{\mathrm{WT}} / \mathrm{RIPK} 3^{\mathrm{KO}}$ and Casp- $8^{\mathrm{ECKO}} / \mathrm{RIPK} 3^{\mathrm{KO}}$ mice. (H and I) Quantitative analysis showing no differences in vessel area (H; $n=11$ WT, $n=7$ ECKO) and number of branches (I; $n=9$ WT, $n=7$ ECKO). Data from 4 independent litters. (J) Working model summarizing the role of Casp-8 as a modulator of angiogenesis. Casp-8 inhibits RIPK3 to allow the proper response of ECs to VEGF stimulation (Casp- $8^{\mathrm{WT}}$ ). If Casp-8 is absent (Casp- $8^{\mathrm{ECKO}}$ ), increased RIPK3 levels induce p38 hyperphosphorylation, which in turn leads to an impaired response to VEGF stimulation and reduced angiogenesis. For $\mathbf{B}-\mathbf{D}, \mathbf{E}, \mathbf{H}$, and $\mathbf{I}$, data are shown as mean $\pm \mathrm{SEM}$. ${ }^{*} P<0.05$; ${ }^{* *} P<0.001,1$-sample $t$ test $(\mathbf{B}$ and C); ${ }^{*} P<0.05 ;{ }^{* *} P<0.01 ;{ }^{* *} P<0.001,2$-way ANOVA with Tukey's multiple comparisons test ( $\mathbf{D}$ and $\left.\mathbf{E}\right) ; 2$-tailed unpaired Student's $t$ test (H and $\left.\mathbf{I}\right)$.

These results indicate that the loss of Casp-8 results in destabilization of EC junctions via an increased basal phosphorylation of p38. They also indicate that the increased basal p38 activity is responsible for the reduced response to VEGF, resulting in overall impaired angiogenesis (see model in Figure 7J).
Activation of 38 MAPK upon loss of Casp- 8 is mediated by RIPK3. Casp-8 inhibits RIPK3 (12), and it has recently been shown that RIPK3 regulates vessel permeability via p38 in ECs (22). Therefore, we asked whether RIPK 3 could act downstream of Casp- 8 to regulate $\mathrm{p} 38$. Interestingly, we observed that Casp $-8^{\mathrm{KD}}$ ECs expressed 
A

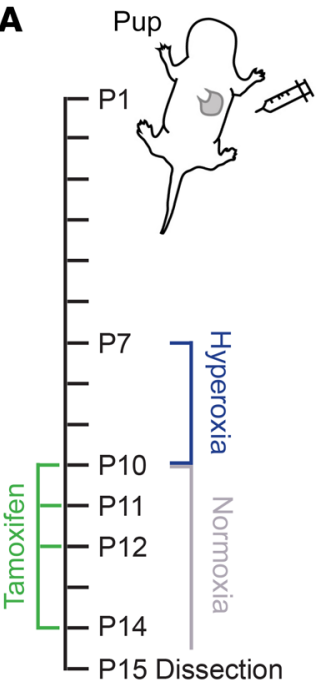

B

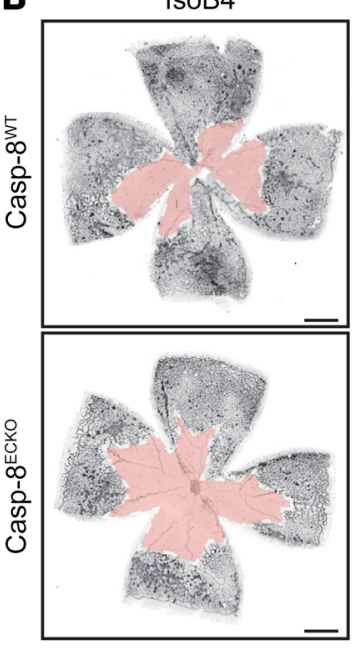

C

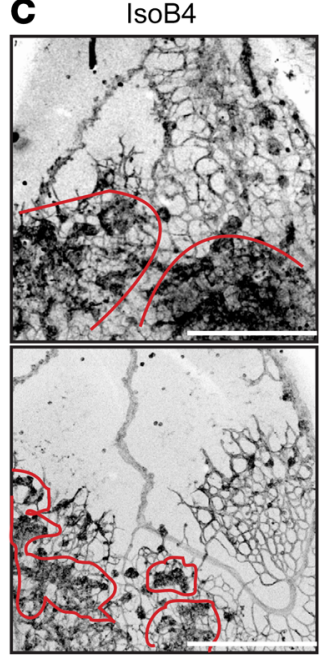

D

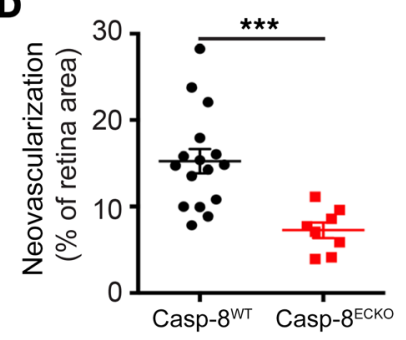

E

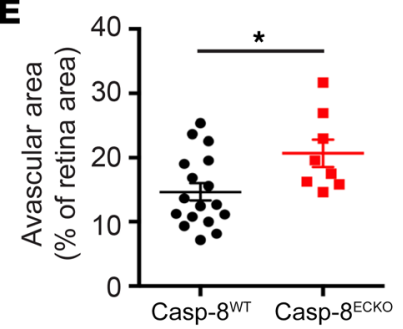

$\mathbf{F}$
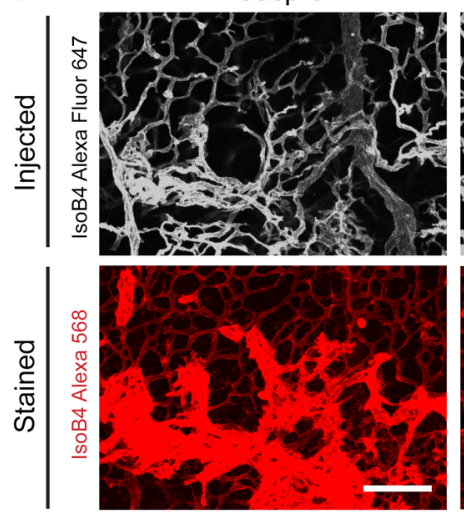

Casp-8

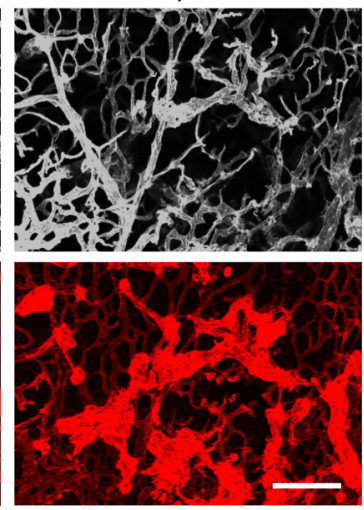

G

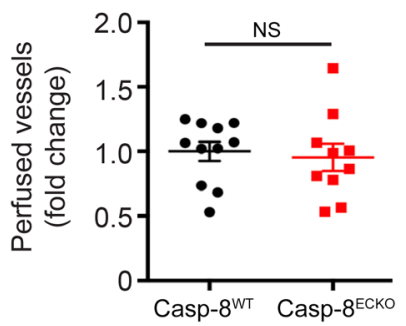

Figure 8. Loss of Casp-8 results in reduced neovascularization in the OIR model. (A) Schematic showing the timeline of the OIR protocol. Pups were placed in 75\% oxygen (hyperoxia) from P7 to P10, then placed back under normal oxygen conditions (normoxia) until P15. Tamoxifen was injected at P10-P12 and P14. (B and C) Representative images of the retinal vasculature stained with IsoB4 in Casp- $8^{\text {WT }}$ and Casp- $8^{\text {ECKO }}$ mice. Red space indicates the avascular area (B), and red outlines indicate neovascular tufts (C). (D and E) Pathological neovascularization is reduced in Casp-8 ${ }^{\text {ECKO }}$ mice (D) compared with Casp- $8^{\text {WT }}$ littermates, while the avascular area was higher in Casp- $8^{\text {ECKO }}$ mice ( $n=16$ WT, $n=8$ ECKO). (F and $\left.\mathbf{G}\right)$ Retroorbital injection of IsoB4-Alexa Fluor 647 did not reveal any difference in vessel perfusion among Casp- $8^{\mathrm{ECKO}}$ and Casp- $8^{\mathrm{WT}}$ mice $(n=11 \mathrm{WT}, n=10$ ECKO), as quantified by measuring Alexa Fluor 647 fluorescent intensity in IsoB4-Alexa Fluor 568-labeled vessels. Data are shown as mean \pm SEM from 3 independent litters. ${ }^{*} P<0.05$; ${ }^{* * *} P<$ 0.001, 2-tailed unpaired Student's $t$ test. Scale bars: $500 \mu \mathrm{m}$ (B); $250 \mu \mathrm{m}$ (C); $100 \mu \mathrm{m}$ (F).

higher RIPK3 protein (Figure 7A) and mRNA levels (Figure 7C), as was also shown in epithelial Casp- $8^{\mathrm{KO}}$ mice (46). To investigate whether $\mathrm{p} 38$ was activated downstream of RIPK3 in Casp- $8^{\mathrm{KD}} \mathrm{ECs}$, we additionally knocked down RIPK3 and analyzed p38 phosphorylation. KD of RIPK3 alone had no impact on either basal p-p38 or on VEGF-induced p38 phosphorylation (Supplemental Figure 7, A and B). However, the basal increase in p-p38 present in Casp- $8^{\mathrm{KD}}$ ECs was rescued to control levels in Casp- $8^{\mathrm{KD}} / \mathrm{RIPK}^{\mathrm{KD}} \mathrm{ECs}$ (Figure 7, $A$ and $B$ ), indicating that RIPK 3 was responsible for the increased p-p38 levels observed in Casp- $8^{\mathrm{KD}}$ cells.

Consistent with these effects on p-p38, KD of RIPK3 also rescued the ability of Casp- $8^{\mathrm{KD}} \mathrm{EC}$ s to respond to VEGF and to form tube-like structures to an extent similar to that seen for Casp- $8^{\mathrm{WT}}$ and RIPK3 ${ }^{\mathrm{KD}}$ ECs (Figure 7D and Supplemental Figure $7 \mathrm{C}$ ). In addition, proliferation induced by VEGF or FGF in Casp- $8^{\mathrm{KD}}$ RIPK3 ${ }^{\mathrm{KD}}$ ECs was also partially rescued compared with that in Casp- $8^{\mathrm{KD}}$ ECs, as measured by BrdU incorporation (Figure 7E).

To confirm the role of RIPK3 in Casp- $8^{\mathrm{ECKO}}$ mice in vivo, we crossed our Cdh5-(PAC)-CreERT $2 \times$ Casp- $8^{\mathrm{A} / \mathrm{l}}$ mice with a RIPK $3^{\mathrm{KO}}$ mouse line (47). In line with our in vitro results, RIPK3 did not regulate angiogenesis under physiological conditions, as neither the vessel area nor the number of branchpoints (Supplemental Figure 7, D-G) in the retina of heterozygous or homozygous RIPK3 ${ }^{\mathrm{KO}}$ pups was affected at P6 compared with those of WT littermates. In contrast, the vascular defects in Casp- $8^{\mathrm{ECKO}}$ mice were rescued in Casp-8 ${ }^{\text {ЕСКо }} / \mathrm{RIPK}^{\text {Ко }}$ mice, as the vessel area (Figure 7, F-H) and number of branchpoints (Figure 7I) were similar to those of Casp- $8^{\mathrm{WT}} / \mathrm{RIPK}^{\mathrm{KO}}$ mice.

In summary, these results show that, in the absence of Casp-8, RIPK3 mediates increased phosphorylation of p38 in vitro and impaired developmental vascular growth in vivo. We propose 
that this RIPK3/p-p38 axis deregulates EC behavior, resulting in impaired angiogenesis (Figure 7J).

Casp- $8^{E C K O}$ mice show reduced pathological angiogenesis in a model of ROP. So far, our results indicate that Casp-8 is required for proper developmental angiogenesis and, when absent, EC proliferation, migration, and cell junction formation do not occur properly, resulting in reduced vessel sprouting and growth. To analyze the impact of these vascular defects in Casp- $8^{\mathrm{ECKO}}$ mice at later developmental stages, we extended our tamoxifen treatment protocol to the first 2 postnatal weeks and analyzed Casp- ${ }^{\mathrm{ECKO}}$ mice at P15 and P42 (Supplemental Figure 8). At both stages, Casp- ${ }^{\mathrm{ECKO}}$ mice did not show differences in the vessel area (Supplemental Figure 8, B and E) or number of branches (Supplemental Figure 8, C and F) compared with control littermates. In addition, we analyzed vessel integrity by injecting $70 \mathrm{kDa}$ fluorescently labeled dextran and could not detect any obvious difference in vessel permeability (Supplemental Figure 8 , D and G) between genotypes. Taken together, these data suggest that compensatory mechanisms overcome the loss of Casp-8 in ECs while mouse development continues.

Despite the better control of oxygen administration to preterm infants, ROP persists in extremely low gestational ages and birth weights and is still a clinical problem (48). ROP is characterized by 2 phases. In phase 1, exposure of preterm infants to high extrauterine oxygen levels causes cessation of vessel growth. However, as the metabolic demand of the retina increases over time and as there is a lack of proper tissue oxygenation, upregulation of VEGF induces pathological neovascularization, consisting of extensive extraretinal neovascular tufts in phase 2 of ROP (49). As our in vitro data showed that Casp- $8^{\mathrm{KD}}$ ECs were not able to respond to VEGF, we explored whether the loss of Casp-8 could also reduce pathological angiogenesis during ROP. We therefore applied the OIR model, which closely resembles the 2 phases of ROP $(50,51)$. In this model, high oxygen levels induce rapid vessel regression, followed by upregulation of VEGF, which causes pathological vessel sprouting and formation of abnormal and leaky vascular tufts.

For this, we exposed pups to high oxygen levels (75\%) to induce vessel regression from P7 to P10 (Figure 8A), as previously described (35). Subsequently, pups were returned to normoxia until P15, causing the now avascular retina to become hypoxic and upregulate VEGF, inducing the neovascular response. Cre recombination and Casp-8 deletion were induced on return to normoxia from P10 to P14 to evaluate the effect of Casp- 8 loss on pathological neovascularization (Figure 8A). Analysis of the retinal vasculature at P15 showed that pathological neovascularization (quantified by measuring the neovascular tuft area; ref. 52) was strongly reduced in Casp- $8^{\mathrm{ECKO}}$ compared with Casp- $8^{\mathrm{WT}}$ retinas (Figure 8, $\mathrm{C}$ and D), supporting the concept that blocking Casp-8 is beneficial for preventing disease progression. In addition to pathological neovascularization, normal angiogenesis gradually replaced the vessels lost from the central retina following exposure to high oxygen. Analysis of the avascular area showed a slight increase in Casp- $8^{\mathrm{ECKO}}$ pups compared with WT littermates (Figure 8, B and E). This result is probably a combination of reduced pathological neovascularization and reduced vessel regrowth, consistent with our finding that angiogenesis and the response to VEGF were reduced in Casp- $8^{\mathrm{KD}}$ ECs.
Finally, to examine vessel integrity and functionality of the newly formed vasculature, we analyzed vessel perfusion by retroorbital injection of IsoB4-Alexa Fluor 647 (which attaches to the vessel lumen and hence, labels perfused vessels), as previously described $(53,54)$. Then the isolated retinas were costained with IsoB4-Alexa Fluor 568 to determine the vessel area. Analysis of IsoB4-Alexa Fluor 647 per vessel area revealed efficient vessel perfusion in both genotypes (Figure $8, \mathrm{~F}$ and $\mathrm{G}$ ).

Taking these data together, we determined that targeting Casp-8 in ECs in a model of OIR, resembling ROP, could indeed be beneficial, as it reduces the severity of tuft formation without compromising the newly formed vasculature.

\section{Discussion}

Components of both the intrinsic and extrinsic apoptosis signaling pathways have been described as being active in ECs and involved in different processes during the formation of the vascular system. While the intrinsic pathway of apoptosis (induced by the activation of Bax and Bak) is crucial for the regression and cell death of ECs from hyaloid vessels in the retina (55-58), this pathway controls EC numbers $(8,10,59-61)$, but is not an active driver of vessel remodeling/pruning in the postnatal retina vasculature. On the other hand, activation of the extrinsic signaling machinery via activation of CD95 expressed in retinal ECs contributes to vessel development by regulating EC proliferation instead of cell death (62).

Casp-8 is a key protease in the extrinsic cell death signaling pathway that has both cell death-inducing and prosurvival functions (21). A basal Casp-8 activity is required for cell survival, as it inhibits the activation of RIPK3 and thus the subsequent phosphorylation of MLKL, leading to necroptosis (63). Under pathological conditions, tumor-induced EC necroptosis has been described as a mechanism via which tumor cells extravasate and metastasize (23). However, in the same tumor model, it has also been shown that RIPK3 regulates vessel permeability independently of EC necroptosis (22). Here, we show that necroptosis is not involved in postnatal developmental angiogenesis, as the vascular defects in Casp- $8^{\text {ЕСКО }}$ mice are still present in Casp- $8^{\text {ЕСКО }} / \mathrm{MLKL}^{\mathrm{KO}}$ retinas. Notably, proper angiogenesis was restored in Casp- $8^{\text {ЕСКО/ }}$ RIPK $3^{\mathrm{KO}}$ pups, indicating that the vascular impairments in Casp- $8^{\mathrm{ECKO}}$ mice were mediated by RIPK3, thus assigning a necroptosisindependent function for RIPK3 in ECs during angiogenesis.

Cell death-independent functions of Casp- 8 have been shown to be both activity dependent $(16,64)$ and independent (acting as a scaffold protein; refs. 14, 32). While we do not rule out that Casp- 8 could also act as a scaffold protein to regulate the angiogenic response of ECs, here we show that its activity is required. The cellular substrate for Casp-8 in ECs during angiogenesis has yet to be identified. However, as RIPK3 mRNA levels are upregulated in Casp- $8^{\mathrm{KD}} \mathrm{ECs}$, which is similar to what occurs in epithelial cells without Casp-8 (46), transcriptional regulators might be involved.

Mechanistically, Casp-8 deletion in ECs results in EC proliferation, sprouting, and VE-cadherin subcellular distribution defects. Analysis of different signaling pathways revealed that Casp- $8^{\mathrm{KD}}$ ECs had increased basal activation of p38. Consistent with our findings, loss of Casp-8 in the epidermis has also been shown to induce the upregulation of p-p38 (65). Despite the fact that VEGF 
stimulation activated ERK, Akt, FAK, and p38 in Casp- $8^{\mathrm{KD}}$ ECs, this did not result in a functional angiogenic response in vitro. However, when p38 activity was blocked, Casp- $8^{\mathrm{KD}}$ ECs responded normally to VEGF stimulation in the sprouting and tube-formation assay. These findings indicate that the basal overactivation of p38 was already enough to functionally inhibit the response of Casp- $8^{\mathrm{KD}}$ ECs to VEGF and thus to impair angiogenesis. It was previously reported that p38 activity induces EC migration (66). However, in our experimental setup, EC migration was not affected in Casp- ${ }^{\mathrm{KD}}$ ECs under basal conditions, even though p38 was already more highly activated. As migration of Casp- $8^{\mathrm{KD}}$ ECs does not change under basal conditions, this suggests that p38-independent mechanisms might account for this phenotype. We could also link RIPK3 to p38 hyperactivation, as in vitro KD of RIPK3 in Casp $-8^{\mathrm{KD}}$ ECs rescued the basal phosphorylation of $\mathrm{p} 38$ to control levels. Further studies are needed to investigate how RIPK3 regulates p38 and whether RIPK3 activity is required.

Specific loss of Casp-8 in the epidermis results in increased p38 phosphorylation (65) and loss of junction integrity in epithelial cells, independently of cell death (40). Here, we show that loss of Casp-8 in ECs in vitro resulted in impaired distribution of VE-cadherin at the cell membrane, creating a serrated pattern. Moreover, Casp- $8^{\mathrm{KD}}$ ECs failed to induce VE-cadherin rearrangements required for proper angiogenesis upon VEGF stimulation. This phenotype was rescued when p38 was inhibited in vitro. So far, it is not very well understood how p38 regulates VE-cadherin localization under physiological conditions. However, tumor transendothelial cell migration studies suggest that p38 does not act directly on VE-cadherin, but rather promotes its internalization via the formation of stress fibers (67). We therefore hypothesize that the activation of $\mathrm{p} 38$ in the absence of Casp- 8 could cause the VE-cadherin defects via a similar mechanism.

In vivo, VE-cadherin stability was altered at the back of the retina of Casp- $8^{\mathrm{ECKO}} \mathrm{EC}$ pups, suggesting that EC-EC contacts fail to stabilize. Recent studies indicate that, rather than its sole presence or absence $(35-37,39)$, it is the dynamic and heterogeneous expression of VE-cadherin in different areas of the developing vessel sprout that drives angiogenesis (38). Consistent with this current view, which suggests that EC junctions in the back of the retina have to be stabilized in order to allow proper sprout elongation in the front, we postulate that the increased number of vessel patches with serrated junctions in the back of Casp- $8^{\mathrm{ECKO}}$ retinas blocks EC positional interchanges, ultimately resulting in a reduced number of sprouts at the vascular front and thus reduced angiogenesis.

ROP in preterm infants is characterized by pathological retina neovascularization and excessive extraretinal tuft formation (50, 68). Here, by applying the OIR model, resembling ROP, we show that Casp-8 is mechanistically involved in the pathophysiology of developmental neovascular ocular disease. Indeed, Casp- $8^{\text {Еско }}$ mice, in which Casp-8 was deleted in ECs just during the neovascularization phase, presented reduced aberrant vascular tufts. Consistent with our findings that Casp- $8^{\mathrm{KD}}$ ECs have a reduced response to VEGF stimulation and thus show reduced VEGFinduced angiogenesis, we also observed a slight increase in the avascular area, suggesting that the newly regrowing vessels develop more slowly in Casp- $8^{\mathrm{ECKO}}$ retinas compared with Casp- $8^{\text {WT }}$ retinas (however, we cannot exclude that in this specific setting, necroptosis contributes to the increased avascular area observed

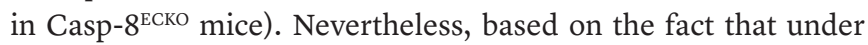
physiological conditions, Casp- $8^{\mathrm{ECКO}}$ retinas finally achieve a normal and functional vasculature, we propose that inactivation of Casp-8 in ECs during ROP is beneficial, as it will inhibit aberrant neovascularization, and in a way similar to that seen in development, the avascular area will be finally recovered. Whether targeting Casp-8 is also beneficial in an adult setting of ischemic retinopathy, such as occurs during proliferative diabetic retinopathy or retinal vein occlusion, requires further investigation. Although we have shown that the loss of Casp-8 in ECs is compensated during development and that Casp- $8^{\mathrm{ECKO}}$ mice at P15 and P42 did not present vascular defects or increased vessel permeability, we cannot rule out that the acute deletion of Casp- 8 in ECs in the healthy or diseased adult retinal vasculature might have a negative impact. In addition, even though the results presented in the OIR model are encouraging, we acknowledge that further clinical evidence is necessary and should be provided in the future to determine whether and how Casp- 8 could be locally targeted in retina ECs in human patients.

\section{Methods}

Please see Supplemental Methods for further experimental procedures.

Mouse lines and treatment. Casp- $8^{\mathrm{fl} / \mathrm{fl}}$ mice (69) were crossed with Cdh5(PAC)-CreERT2 mice (24) to specifically delete Casp-8 in the endothelium upon tamoxifen treatment. MLKL ${ }^{\mathrm{KO}}$ mice were provided by M. Pasparakis (CECAD, University of Cologne, Cologne, Germany).

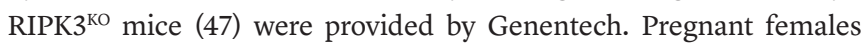
were i.p. injected with $2 \mathrm{mg}$ tamoxifen/30 g body weight from E8.5 until E10.5. Embryos were dissected at E13.5 and assessed for heartbeat (alive), death, or presence of malformations, such as bleedings or (cardiac) edema (Supplemental Figure 1B). Recombination in pups was induced by intragastric injection of $50 \mu \mathrm{L}$ tamoxifen $(1 \mathrm{mg} / \mathrm{mL})$ at P1-P3 and P5 as shown in Figure 1A. For analysis at P15 and P42, pups received additional tamoxifen injections (i.p.) at P8, P11, and P14.

OIR model. To induce retinal pathological angiogenesis in Casp- $8^{\text {ЕСКо }}$ mice, the OIR model was used, as previously described (35). From P7 to $\mathrm{P} 10$, pups were placed in $75 \%$ oxygen (hyperoxia); at P10, pups were returned to normal oxygen conditions (normoxia) until P15 (35). Cre recombination was induced by i.p. injection of $50 \mu \mathrm{L}$ tamoxifen $(2 \mathrm{mg} /$ $\mathrm{mL}$ ) at P10-P12 and P14. To analyze vessel perfusion, pups at P15 were retroorbitally injected with IsoB4-Alexa Fluor 647 (5 mg/kg body weight), as previously described (54), and sacrificed after 5 minutes. Retinas were isolated and fixed in 4\% PFA/PBS for 1 hour at room temperature (RT). Conditions used for the retina staining are specified in the Retina dissection, processing, and staining section. Quantification of the size of the avascular area and neovascular tufts was performed in Adobe Photoshop with investigators blinded to experimental conditions, as previously described (52). To quantify vessel perfusion, mean fluorescent intensity of injected IsoB4-Alexa Fluor 647 was normalized to the intensity of vascular stained with IsoB4-Alexa Fluor 568, as previously described (54).

Analysis of EC proliferation in vivo. EdU (Thermo Scientific) was injected at a concentration of $100 \mu \mathrm{g} / \mathrm{g}$ body weight into P6 Casp$8^{\text {EСКО }}$ pups, as previously described (70), 2.5 hours before culling. Eyes were fixed in $4 \% \mathrm{PFA}$ for 1.5 hours at $4^{\circ} \mathrm{C}$. EdU $\mathrm{E}^{+}$cells in the retina were detected with the Click-iT EdU Alexa Fluor 488 Imaging Kit 
(Thermo Scientific), according to the manufacturer's instructions. ECs were counterstained with ERG and IsoB4. Eight pictures per pup of the angiogenic front were imaged at $\times 20$ magnification with an LSM 510 META. Quantification was done with investigators blinded to experimental conditions. The number of proliferating ECs is expressed as $\mathrm{EdU}^{+} \mathrm{ERG}^{+}$cells normalized to the IsoB $4^{+}$vessel area per field of view.

Whole-mount staining of the yolk sac vasculature. Yolk sacs were fixed overnight in $4 \% \mathrm{PFA} / \mathrm{PBS}$ at $4^{\circ} \mathrm{C}$. After permeabilization in $1 \%$ Triton X-100/PBS for 1 hour at RT and blocking in 0.2\% BSA, 5\% normal donkey serum (Dianova), and 0.3\% Triton X-100 in PBS for 2 hours at RT, primary antibody Endoglin (1:200, MAB1320, R\&D Systems) was incubated overnight at $4^{\circ} \mathrm{C}$ in blocking solution. The appropriate Alexa Fluor-conjugated secondary antibody was incubated overnight at $4^{\circ} \mathrm{C}$. Whole-mount images were acquired on an LSM 510 META confocal microscope.

Retina dissection, processing, and staining. Eyes were collected and enucleated in PBS. For VE-cadherin staining, eyes were fixed in $2 \%$ PFA/PBS for 1 hour at $4^{\circ} \mathrm{C}$. For all other staining, eyes were fixed for 1.5 hours in $4 \% \mathrm{PFA} / \mathrm{PBS}$ at $4^{\circ} \mathrm{C}$. Retinas were dissected, permeabilized with PBS containing $1 \%$ BSA and $0.3 \%$ Triton X-100 for 1 hour, and incubated with primary antibodies at $4^{\circ} \mathrm{C}$ overnight. Antibodies used were as follows: Alexa Fluor 594-conjugated isolectin GS-IB4 (1:250, catalog I21413, Thermo Scientific), Casp-8 (1:100, catalog 4927, Cell Signaling Technology), ERG (1:200, catalog ab92513, Abcam), collagen IV (1:200, catalog CO20451, BIOZOL), Desmin (1:200, catalog ab15200, Abcam), VE-cadherin (1:100, catalog 555289, BD Biosciences), claudin-5 (1:100, catalog 34-1600, Thermo Scientific), and cCasp-3 (1:100, catalog 661, Cell Signaling). After washing with PBS, retinas were incubated for 2 hours at RT with the respective secondary antibody. TUNEL assay (Roche) was performed according to the manufacturer's instructions. Briefly, retinas were fixed for 2 hours in $4 \% \mathrm{PFA} / \mathrm{PBS}$ at RT. Permeabilization was done in $0.5 \%$ Triton $\mathrm{X}-100 / 0.1 \%$ citrate in PBS for 3 hours at RT. Then labeling of TUNEL ${ }^{+}$ cells was done for 1 hour at $37^{\circ} \mathrm{C}$. After washing, samples were stained with FITC-conjugated IsoB4 (1:250, ALX-650-001F-MC05, Enzo Life Sciences) in 1\% BSA, 0.3\% Triton X-100/PBS, for 1 hour at RT. Retinas were flat mounted and analyzed using a confocal fluorescence microscope (LSM 510 META or LSM800; Carl Zeiss).

Analysis of VE-cadherin localization in ECs in vivo. For analysis of VE-cadherin in the retina of P6 pups, high-magnification pictures $(\times 63)$ were acquired. Two pictures from either the angiogenic front or the back of the retina were randomly taken in areas adjacent either to a vein or an artery. Representative inset pictures show the most active or inhibited pattern of VE-cadherin in Casp- $8^{\mathrm{WT}}$ and Casp- $8^{\mathrm{ECKO}}$ pups. Quantification of the percentage of VE-cadherin patches was done with investigators blinded to experimental conditions using MATLAB as previously described (35).

ISH in the retina. ISH was performed with minor modifications, as previously described (71). In brief, eyes of P6 pups were removed and fixed for 20 minutes in $4 \%$ PFA on ice. After isolation and dissection, retinas were stored in methanol at $-20^{\circ} \mathrm{C}$ overnight. Hybridization was performed at $66^{\circ} \mathrm{C}$. Primer sequences to generate probes to detect mouse Casp- 8 were as follows: Casp-8, forward: 3'-TTTCCACATCAGTCGGTGGG-5'; Casp-8, reverse: 3'-CTCTTGGCGAGTCACACAGT-5'. After color development, retinas were extensively washed with PBS and counterstained with Alexa Fluor 594-conjugated IsoB4. Bright field and fluorescent images were acquired simultaneously using a Zeiss LSM 800 ( $\times 20$ objective). Bright field images were processed and background corrected with a rolling ball algorithm in Image $(\mathrm{NIH})$.

Lung EC isolation. Murine lung ECs were isolated by MACS Technology (Miltenyi Biotec). Lungs of P6 mouse pups were isolated and kept in ice-cold HBSS containing $10 \%$ FCS. Tissue from Casp- $8^{\text {wT }}$

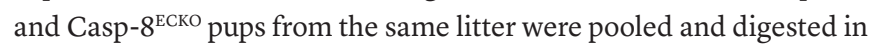
collagenase I/DNAse I $(0.1 \mathrm{mg} / \mathrm{mL}$, Roche $)$ at $37^{\circ} \mathrm{C}$. The digested tissue was filtered, incubated with Red Blood Cell Lysis Buffer (Roche), and washed several times with PEB buffer (0.5\% BSA, 2 mM EDTA in PBS). Negative selection of $\mathrm{CD} 45^{+}$cells was performed by incubation of the cell suspension with CD45 MicroBeads (1:10) for 15 minutes at $4^{\circ} \mathrm{C}$. Cell suspensions were applied onto LS columns, and unlabeled cells were collected on ice. Enrichment of ECs was performed using CD31 MicroBeads (1:10) in PEB buffer for 15 minutes at $4^{\circ} \mathrm{C}$ and application on MS columns. The positive cell fraction was eluted from the columns with PEB buffer and directly frozen in RLT buffer (QIAGEN) for RNA isolation.

Analysis of VE-cadherin localization in ECs in vitro. For VE-cadherin staining in HUVECs, $1 \times 10^{5}$ infected cells (shCtrl or shCasp-8) were seeded and allowed to attach overnight. Cells were starved overnight and stimulated with $50 \mathrm{ng} / \mathrm{mL}$ VEGF for the indicated time points. After fixation in 4\% PFA/PBS for 30 minutes at RT and permeabilization in $0.5 \%$ Triton X-100/PBS for 10 minutes, blocking in 1\% BSA, $20 \%$ normal donkey serum (Dianova) in 0.2\% Triton X-100/PBS was done for 1 hour at RT. Samples were incubated with primary antibody (anti-VE-cadherin, 1:200, catalog 610252, BD Bioscience) for 2 hours at RT, followed by incubation with the appropriate Alexa Fluorconjugated secondary antibody for 2 hours at RT. Nuclei were counterstained with DAPI (1:1000, D1306, Invitrogen). After mounting, random fields of view were imaged with a Zeiss LSM 800 ( $\times 63$ objective). VE-cadherin at the cell perimeter of single, nonadjacent cells was manually traced with ImageJ. Total VE-cadherin at the cell perimeter was calculated as the sum of all individual VE-cadherin patches. At least 15 cells per condition were quantified with investigators blinded to the experimental condition from 3 independent experiments.

Fibrin gel bead sprouting assay. Fibrin gel bead sprouting assay was performed as previously described (72). Briefly, Cytodex 3 microcarrier beads (GE Healthcare) were coated with siRNA-transfected or shRNA-infected HUVECs (mixed at 200 cells per bead) and embedded in $2 \mathrm{mg} / \mathrm{mL}$ fibrin gels ( $2 \mathrm{mg} / \mathrm{mL}$ fibrinogen [Calbiochem], 0.625 units $/ \mathrm{mL}$ thrombin [Sigma-Aldrich], and 0.15 units $/ \mathrm{mL}$ aprotinin [Sigma-Aldrich]). Beads were cultured in 2\% FBS growth factor-free Endopan 3 medium in the presence or absence of $50 \mathrm{ng} / \mathrm{ml}$ VEGF. After 24 hours, the culture was fixed with 4\% PFA for 15 minutes, blocked in 1\% BSA, $0.2 \%$ Triton X-100-PBS, and incubated with 1 $\mu \mathrm{g} / \mathrm{mL}$ phalloidin-fluo (94507, Sigma-Aldrich) for 2 hours. Confocal images were taken with a Zeiss LSM 510 microscope and analyzed by ImageJ. Quantification was done with investigators blinded to experimental conditions. Approximately 20 beads per condition were quantified from 4 independent experiments.

Single EC tracings. Single EC tracings were performed as previously described (33). Infected ECs with shRNA control or shCasp-8 were plated on 6-well plates and stimulated with $50 \mathrm{ng} / \mathrm{mL}$ VEGF or control vehicle (after overnight starvation). Phase contrast images of 10 fields of view per well were acquired every 10 minutes over the course of 12 hours using an inverted NikonTi microscope (with a Nikon Plan 
Fluor $\times 10$ NA 0.3 objective) equipped with an environmental box from Oko Lab for temperature, $\mathrm{CO}_{2}$, and humidity control. EC migration was traced automatically using NIS Elements 4.5 software. At least 30 cells per condition were quantified with investigators blinded to experimental conditions from 3 independent experiments.

Tube formation. Tube-formation assays were performed in $\mu$-Slide Angiogenesis wells (ibidi $\mathrm{GmbH}$ ) as previously described $(33,73)$ using $10 \mu \mathrm{L}$ of growth factor-reduced Matrigel (BD Biosciences) per well. In brief, siRNA-transfected or lentivirus-infected HUVECs resuspended in 2\% FBS growth factor-free Endopan 3 medium, with or without VEGF $(50 \mathrm{ng} / \mathrm{mL}$ ), were seeded onto the polymerized Matrigel. Wherever indicated, prior to seeding on Matrigel, cells were pretreated overnight with SB203580 $(1 \mu \mathrm{M})$. Cells were incubated for 4 hours in a humidified chamber at $37^{\circ} \mathrm{C}, 5 \% \mathrm{CO}_{2}$ before analysis. Images were acquired with a wide-field microscope (Zeiss Axiovert 200) equipped with an Axiocam MRm camera and $\mathrm{a} \times 5$ objective. At least 3 fields of view per condition were acquired. Quantification of 3 independent experiments was done with investigators blinded to experimental conditions using the Angiogenesis analyzer tool of ImageJ.

Scratch assay. siRNA-transfected HUVECs were seeded in a 6-well plate. When cells were confluent, they were starved in $2 \%$ FBS growth factor-free Endopan 3 medium overnight. A wound was induced by scraping the cell monolayer with a P200 pipette tip, and cells were stimulated with or without VEGF $(50 \mathrm{ng} / \mathrm{mL})$. A picture was acquired at time-point zero and 12 hours after incubation at $37^{\circ} \mathrm{C}$. The percentage of wound closure between 0 hours and 12 hours of VEGF stimulation was analyzed with ImageJ with investigators blinded to experimental conditions. Results are from at least 3 independent experiments. For each treatment, 8 to 10 fields of view were analyzed.

In vitro cell death assay. Infected HUVECs $\left(1 \times 10^{5}\right.$ cells/well in 6-well plates) were treated with TNF- $\alpha(100 \mathrm{ng} / \mathrm{mL}$, Preprotech) and TRAIL (100 ng/mL, made in house) in normoxia or hypoxia $(0,1 \%$ $\mathrm{O}_{2}$, BioSpherix, X2, Exvivo System) during 24 hours. After treatment, cells were washed with PBS and stained with PI $(40 \mu \mathrm{g} / \mathrm{ml})$ while being treated with RNase $(100 \mu \mathrm{g} / \mathrm{ml})$. Quantitative analysis of $\mathrm{PI}^{+}$cells was carried out in a FACSCalibur cytometer using Cell Quest software (BD).

Casp-8 Glo assay. The Casp-8 Glo assay was performed in combination with the CellTiter-Fluor Cell Viability Assay (both from Promega) according to the manufacturer's instructions. In brief, $6 \times 10^{3}$ HUVECs per well were seeded in a 96-well plate. After overnight starvation, cells were treated with Z-IETD-FMK $(10 \mu \mathrm{M})$ or TNF $(100 \mathrm{ng} / \mathrm{mL})+\mathrm{CHX}$ $(10 \mu \mathrm{g} / \mathrm{mL})$ for 4 hours before cell viability and Casp-8 activity were measured. Data are expressed as relative units (RU) resulting from the Casp-8 Glo (relative luminescent units [RLU])/cell viability (relative fluorescence units [RFU]) ratio.

Statistics. Results are expressed as mean \pm SEM. To calculate statistical significance, 2-tailed Student's $t$ test, 1-sample $t$ test, 1-way ANOVA, or 2-way ANOVA followed by Bonferroni's multiple comparisons test was used, as indicated in the figure legends.
To calculate statistical significance for Figure 4 (analysis of VE-cadherin distribution in vivo), the Dirichlet regression model was applied for the analysis of binned data. Additional Mann-Whitney $U$ tests for each state are reported to indicate states with strong differences between groups. All calculations were performed using Prism software. A $P$ value of less than or equal to 0.05 was considered significant.

Study approval. All animal procedures were conducted in accordance with European, national, and institutional guidelines. Protocols were approved by local government authorities (Regierungspräsidium Karlsruhe, Germany).

\section{Author contributions}

NT, AFV, RY, and CRDA conceptualized the study. NT, AFV, RY, and CRDA developed methodology. NT, AFV, RY, IP, SLP, XW, RMP, LC, WWLW, LC, BS, HJG, TH, CM, PH, and CRDA performed or interpreted experiments. CRDA, HGA, and ALR provided resources. NT, AFV, RY, TS, and CRDA wrote the original draft, and NT, AFV, RY, IP, SLP, XW, RMP, WWLW, LC, BS, HJG, LCW, MM, ALR, TS, HGA, TH, CM, PH, RA, and CRDA reviewed and edited the paper. CRDA, ALR, TS, and HGA supervised the study. CRDA acquired funds and administered the project. RA provided mice. The order of first authors was arranged according to their involvement in the revision of the study.

\section{Acknowledgments}

We thank Manolis Pasparakis for providing MLKL ${ }^{\mathrm{KO}}$ mice and Stephen Hedrick and the UCSD for providing Casp- $8^{\mathrm{fl} / \mathrm{l}}$ mice. We thank Bruno Köhler and Genentech for providing the RIPK3 ${ }^{\mathrm{KO}}$ mice. We thank Katie Bentley for her help with the MATLAB image analysis for VE-cadherin staining in vivo. We thank the Nikon imaging Center of the University of Heidelberg for their support. We thank Heike Adler and Melanie Richter for technical assistance and the Ruiz de Almodovar lab for useful discussions. NT was supported by a Heidelberg Biosciences International Graduate School (HBIGS) PhD fellowship; IP was supported by Becas Chile. XW was supported by an Alexander Von Humboldt postdoctoral fellowship. TS is supported by Deutsche Forschungsgemeinschaft (DFG) SCHM 2560/3-1. CRDA is supported by DFG grant RU 1990/ 1-1, ERC (ERC-StG-311367), and DFG SFB873, FOR2325. CRDA, RHA and HGA are supported by DFG grants from SFB1366 (project number 394046768-SFB 1366). LC is supported by a National Health and Research Council (NHMRC) project grant (1125536) and the L.E.W. Carty Charitable Fund.

Address correspondence to: Carmen Ruiz de Almodóvar, Medical Faculty Mannheim of the University of Heidelberg, Ludolf-Krehl Street13-17, 68167 Mannheim, Germany. Phone:49621/38371624; Email: carmen.ruizdealmodovar@medma.uni-heidelberg.de.
1. Sapieha P, et al. Retinopathy of prematurity: understanding ischemic retinal vasculopathies at an extreme of life. J Clin Invest. 2010;120(9):3022-3032.

2. Mutlu FM, Sarici SU. Treatment of retinopathy of prematurity: a review of conventional and promising new therapeutic options. Int JOphthalmol.
2013;6(2):228-236.

3. Potente M, Gerhardt H, Carmeliet P. Basic and therapeutic aspects of angiogenesis. Cell. 2011;146(6):873-887.

4. Coultas L, Chawengsaksophak K, Rossant J. Endothelial cells and VEGF in vascular development. Nature. 2005;438(7070):937-945.
5. Korn C, Augustin HG. Mechanisms of vessel pruning and regression. Dev Cell. 2015;34(1):5-17.

6. Wietecha MS, Cerny WL, DiPietro LA. Mechanisms of vessel regression: toward an understanding of the resolution of angiogenesis. Curr Top Microbiol Immunol. 2013;367:3-32.

7. Kochhan E, et al. Blood flow changes coincide 
with cellular rearrangements during blood vessel pruning in zebrafish embryos. PLoS ONE. 2013;8(10):e75060.

8. Korn C, et al. Endothelial cell-derived non-canonical Wnt ligands control vascular pruning in angiogenesis. Development. 2014;141(8):1757-1766.

9. Cheng C, et al. Endothelial cell-specific FGD5 involvement in vascular pruning defines neovessel fate in mice. Circulation. 2012;125(25):3142-3158.

10. Watson EC, et al. Apoptosis regulates endothelial cell number and capillary vessel diameter but not vessel regression during retinal angiogenesis. Development. 2016;143(16):2973-2982.

11. Hughes MA, et al. Co-operative and hierarchical binding of c-FLIP and caspase-8: a unified model defines how c-FLIP isoforms differentially control cell fate. Mol Cell. 2016;61(6):834-849.

12. Oberst A, et al. Catalytic activity of the caspase8-FLIP(L) complex inhibits RIPK3-dependent necrosis. Nature. 2011;471(7338):363-367.

13. Sun L, et al. Mixed lineage kinase domain-like protein mediates necrosis signaling downstream of RIP3 kinase. Cell. 2012;148(1-2):213-227.

14. Barbero S, Barilà D, Mielgo A, Stagni V, Clair K, Stupack D. Identification of a critical tyrosine residue in caspase 8 that promotes cell migration. J Biol Chem. 2008;283(19):13031-13034.

15. Scharner D, et al. Caspase-8 is involved in neovascularization-promoting progenitor cell functions. Arterioscler Thromb Vasc Biol. 2009;29(4):571-578.

16. Liccardi G, et al. RIPK1 and caspase-8 ensure chromosome stability independently of their role in cell death and inflammation. Mol Cell. 2019;73(3):413-428.e7.

17. Boege $\mathrm{Y}$, et al. A dual role of caspase-8 in triggering and sensing proliferation-associated DNA damage, a key determinant of liver cancer development. Cancer Cell. 2017;32(3):342-359.e10.

18. Varfolomeev EE, et al. Targeted disruption of the mouse Caspase 8 gene ablates cell death induction by the TNF receptors, Fas/Apo1, and DR3 and is lethal prenatally. Immunity. 1998;9(2):267-276.

19. Yeh WC, et al. Requirement for Casper (c-FLIP) in regulation of death receptor-induced apoptosis and embryonic development. Immunity. 2000;12(6):633-642.

20. Sakamaki K, et al. Ex vivo whole-embryo culture of caspase-8-deficient embryos normalize their aberrant phenotypes in the developing neural tube and heart. Cell Death Differ. 2002;9(11):1196-1206.

21. Kang TB, et al. Caspase-8 serves both apoptotic and nonapoptotic roles. JImmunol. 2004;173(5):2976-2984.

22. Hänggi K, et al. RIPK1/RIPK3 promotes vascular permeability to allow tumor cell extravasation independent of its necroptotic function. Cell Death Dis. 2017;8(2):e2588.

23. Strilic B, et al. Tumour-cell-induced endothelial cell necroptosis via death receptor 6 promotes metastasis. Nature. 2016;536(7615):215-218.

24. Wang Y, et al. Ephrin-B2 controls VEGF-induced angiogenesis and lymphangiogenesis. Nature. 2010;465(7297):483-486.

25. Savant S, et al. The orphan receptor Tie1 controls angiogenesis and vascular remodeling by differ- entially regulating Tie 2 in tip and stalk cells. Cell Rep. 2015;12(11):1761-1773.

26. Pitulescu ME, Schmidt I, Benedito R, Adams RH. Inducible gene targeting in the neonatal vasculature and analysis of retinal angiogenesis in mice. Nat Protoc. 2010;5(9):1518-1534.

27. Baffert F, et al. Cellular changes in normal blood capillaries undergoing regression after inhibition of VEGF signaling. Am J Physiol Heart Circ Physiol. 2006;290(2):H547-H559.

28. Pietkiewicz S, Schmidt JH, Lavrik IN. Quantification of apoptosis and necroptosis at the single cell level by a combination of imaging flow cytometry with classical annexin V/propidium iodide staining. J Immunol Methods. 2015;423:99-103.

29. Martín-Pérez R, et al. Oncogenic p95HER2/611CTF primes human breast epithelial cells for metabolic stress-induced downregulation of FLIP and activation of TRAIL-R/ Caspase-8-dependent apoptosis. Oncotarget. 2017;8(55):93688-93703.

30. Stone J, et al. Development of retinal vasculature is mediated by hypoxia-induced vascular endothelial growth factor (VEGF) expression by neuroglia. JNeurosci. 1995;15(7 Pt 1):4738-4747.

31. Lenihan CR, Taylor CT. The impact of hypoxia on cell death pathways. Biochem Soc Trans. 2013;41(2):657-663.

32. Barbero $S$, et al. Caspase-8 association with the focal adhesion complex promotes tumor cell migration and metastasis. Cancer Res. 2009;69(9):3755-3763.

33. Wang X, et al. YAP/TAZ orchestrate VEGF signaling during developmental angiogenesis. Dev Cell. 2017;42(5):462-478.e7.

34. Nikolova-Krstevski V, et al. ERG is required for the differentiation of embryonic stem cells along the endothelial lineage. BMC Dev Biol. 2009;9:72.

35. Bentley K, et al. The role of differential VE-cadherin dynamics in cell rearrangement during angiogenesis. Nat Cell Biol. 2014;16(4):309-321.

36. Vitorino P, Meyer T. Modular control of endothelial sheet migration. Genes Dev. 2008;22(23):3268-3281.

37. Sauteur L, et al. Cdh5/VE-cadherin promotes endothelial cell interface elongation via cortical actin polymerization during angiogenic sprouting. Cell Rep. 2014;9(2):504-513.

38. Cao J, et al. Polarized actin and VE-cadherin dynamics regulate junctional remodelling and cell migration during sprouting angiogenesis. Nat Commun. 2017;8(1):2210.

39. Perryn ED, Czirók A, Little CD. Vascular sprout formation entails tissue deformations and VE-cadherin-dependent cell-autonomous motility. Dev Biol. 2008;313(2):545-555.

40. Li C, et al. Development of atopic dermatitis-like skin disease from the chronic loss of epidermal caspase-8. Proc Natl Acad Sci USA. 2010;107(51):22249-22254.

41. Gordon EJ, et al. The endothelial adaptor molecule TSAd is required for VEGF-induced angiogenic sprouting through junctional c-Src activation. Sci Signal. 2016;9(437):ra72.

42. Sun Z, et al. VEGFR2 induces c-Src signaling and vascular permeability in vivo via the adaptor protein TSAd. JExp Med. 2012;209(7):1363-1377.
43. Nwariaku FE, et al. The role of p38 map kinase in tumor necrosis factor-induced redistribution of vascular endothelial cadherin and increased endothelial permeability. Shock. 2002;18(1):82-85.

44. Khanna P, Yunkunis T, Muddana HS, Peng HH, August A, Dong C.p38 MAP kinase is necessary for melanoma-mediated regulation of VE-cadherin disassembly. Am J Physiol, Cell Physiol. 2010;298(5):C1140-C1150.

45. Cuenda A, et al. SB 203580 is a specific inhibitor of a MAP kinase homologue which is stimulated by cellular stresses and interleukin-1. FEBS Lett. 1995;364(2):229-233.

46. Günther C, et al. Caspase-8 regulates TNF- $\alpha$ induced epithelial necroptosis and terminal ileitis. Nature. 2011;477(7364):335-339.

47. Newton K, Sun X, Dixit VM. Kinase RIP3 is dispensable for normal NF-kappa Bs, signaling by the B-cell and T-cell receptors, tumor necrosis factor receptor 1, and Toll-like receptors 2 and 4 . Mol Cell Biol. 2004;24(4):1464-1469.

48. Hellström A, Smith LE, Dammann O. Retinopathy of prematurity. Lancet. 2013;382(9902):1445-1457.

49. Hartnett ME. Pathophysiology and mechanisms of severe retinopathy of prematurity. Ophthalmology. 2015;122(1):200-210.

50. Scott A, Fruttiger M. Oxygen-induced retinopathy: a model for vascular pathology in the retina. Eye (Lond). 2010;24(3):416-421.

51. Kim CB, D'Amore PA, Connor KM. Revisiting the mouse model of oxygen-induced retinopathy. Eye Brain. 2016;8:67-79.

52. Connor KM, et al. Quantification of oxygeninduced retinopathy in the mouse: a model of vessel loss, vessel regrowth and pathological angiogenesis. Nat Protoc. 2009;4(11):1565-1573.

53. Dubrac A, et al. NCK-dependent pericyte migration promotes pathological neovascularization in ischemic retinopathy. Nat Commun. 2018;9(1):3463.

54. Zhang F, et al. The Robo4 cytoplasmic domain is dispensable for vascular permeability and neovascularization. Nat Commun. 2016;7:13517.

55. Mitchell CA, Risau W, Drexler HC. Regression of vessels in the tunica vasculosa lentis is initiated by coordinated endothelial apoptosis: a role for vascular endothelial growth factor as a survival factor for endothelium. Dev Dyn. 1998;213(3):322-333.

56. Hahn P, Lindsten T, Tolentino M, Thompson CB, Bennett J, Dunaief JL. Persistent fetal ocular vasculature in mice deficient in bax and bak. Arch Ophthalmol. 2005;123(6):797-802.

57. Wang S, Park S, Fei P, Sorenson CM. Bim is responsible for the inherent sensitivity of the developing retinal vasculature to hyperoxia. $D e v$ Biol. 2011;349(2):296-309.

58. Koenig MN, et al. Pro-apoptotic BIM is an essential initiator of physiological endothelial cell death independent of regulation by FOXO3. Cell Death Differ. 2014;21(11):1687-1695.

59. Dimmeler S, Zeiher AM. Endothelial cell apoptosis in angiogenesis and vessel regression. Circ Res. 2000;87(6):434-439.

60. Franco CA, et al. Dynamic endothelial cell rearrangements drive developmental vessel regression. PLoS Biol. 2015;13(4):e1002125.

61. Hughes S, Chang-Ling T. Roles of endothelial cell 
migration and apoptosis in vascular remodeling during development of the central nervous system. Microcirculation. 2000;7(5):317-333.

62. Chen S, et al. CNS macrophages control neurovascular development via CD95L. Cell Rep. 2017;19(7):1378-1393.

63. Rodriguez DA, et al. Characterization of RIPK3-mediated phosphorylation of the activation loop of MLKL during necroptosis. Cell Death Differ. 2016;23(1):76-88.

64. O'Donnell MA, et al. Caspase 8 inhibits programmed necrosis by processing CYLD. Nat Cell Biol. 2011;13(12):1437-1442.

65. Lee P, Lee DJ, Chan C, Chen SW, Ch'en I, Jamora C. Dynamic expression of epidermal caspase 8 simulates a wound healing response. Nature.
2009;458(7237):519-523.

66. Rousseau S, Houle F, Landry J, Huot J. p38 MAP kinase activation by vascular endothelial growth factor mediates actin reorganization and cell migration in human endothelial cells. Oncogene. 1997;15(18):2169-2177.

67. Tremblay PL, Auger FA, Huot J. Regulation of transendothelial migration of colon cancer cells by E-selectin-mediated activation of $\mathrm{p} 38$ and ERK MAP kinases. Oncogene. 2006;25(50):6563-6573.

68. Al-Shabrawey M, Elsherbiny M, Nussbaum J, Othman A, Megyerdi S, Tawfik A. Targeting neovascularization in ischemic retinopathy: recent advances. Expert Rev Ophthalmol. 2013;8(3):267-286.

69. Beisner DR, Ch'en IL, Kolla RV, Hoffmann A, Hedrick SM. Cutting edge: innate immunity con- ferred by B cells is regulated by caspase- 8 . JImmunol. 2005;175(6):3469-3473.

70. Neto F, et al. YAP and TAZ regulate adherens junction dynamics and endothelial cell distribution during vascular development. Elife. 2018;7:e31037.

71. Powner MB, Vevis K, McKenzie JA, Gandhi P, Jadeja S, Fruttiger M. Visualization of gene expression in whole mouse retina by in situ hybridization. Nat Protoc. 2012;7(6):1086-1096.

72. Nakatsu MN, Davis J, Hughes CC. Optimized fibrin gel bead assay for the study of angiogenesis. JVis Exp. 2007;(3):e186.

73. Himmels P, et al. Motor neurons control blood vessel patterning in the developing spinal cord. Nat Commun. 2017;8:14583. 